\title{
The Current Understanding of Molecular Pathogenesis of Quantitative von Willebrand Disease, Types 1 and 3
}

\author{
Hamideh Yadegari $^{1}$ Johannes Oldenburg ${ }^{1}$
}

Address for correspondence Prof. Dr. med. Johannes Oldenburg, Institute of Experimental Haematology and Transfusion Medicine, University Clinic Bonn, Sigmund-Freud-Str. 25, Bonn NRW 53127, Germany (e-mail: johannes.oldenburg@ukb.uni-bonn.de).

\section{Abstract \\ Keywords \\ - von Willebrand factor \\ - von Willebrand disease \\ - bleeding disorder \\ - gene mutation}

Zusammenfassung
Von Willebrand disease (VWD), the most prevalent congenital bleeding disorder, arises from deficiencies in quantity or quality of von Willebrand factor (VWF). The quantitative deficiencies of VWF are considered to be either VWD type 1 (mild/moderate reduction of VWF) or type 3 (virtual absence of VWF). Following cloning of the VWF gene (VWF) in the 1980s, significant progress has been made in our understanding of the pathogenesis of VWD. The genetic basis of type 3 VWD is well defined. VWF causative variations comprising predominantly null alleles have been identified in more than $85 \%$ of cases. In contrast, the molecular mechanisms in type 1 disease are only partially characterized. The VWF sequence variations, including mostly missense alterations, are found in only approximately $65 \%$ of type $1 \mathrm{VWD}$ patients. It appears that genetic elements outside of VWF may contribute to the pathophysiology of type 1 VWD. This review discusses in detail the current understandings of the genetic basis and molecular mechanisms causing quantitative deficiencies of VWF.

Die Von Willebrand Erkrankung (VWE) ist die häufigsten erbliche Blutungsstörung, die durch Fehler im von Willebrand Faktor (VWF) verursacht wird. Diese Fehler können entweder zu einer Reduktion der VWF-Konzentration führen oder die Funktion des VWF beeinträchtigen. Wenn die VWF-Konzentration leicht bzw. mäßig reduziert ist, spricht man von einer VWE Typ 1, wenn praktisch kein VWF mehr nachweisbar ist von einer VWE Typ 3. Seit der Klonierung des VWF Gens (VWF) innerhalb der achtziger Jahre hat man viel über die Entstehung der VWE herausgefunden. Die genetische Basis des VWE Typ 3 ist relativ gut erklärt. VWF-verursachende Mutationen, die vorwiegend NullAllele einschließen, wurden in mehr als 85\% der Fälle identifiziert. Im Gegensatz dazu sind die genetischen Ursachen des VWE Typ 1 nur teilweise charakterisiert. Die VWFSequenzvariationen, die hauptsächlich Missense-Mutationen umfassen, werden nur bei ca. $65 \%$ der Patienten mit Typ 1 des VWE gefunden. Die Pathophysiologie der VWE des Typs 1 scheint zusätzlich noch durch genetische Faktoren beeinflusst zu werden, die außerhalb des VWF liegen. In dem hier vorliegenden Review werden ausführlich die genetischen Grundlagen sowie die molekularen Mechanismen diskutiert, die zu einer Reduktion der VWF-Konzentration führen. received

March 16, 2019

accepted after revision

September 26, 2019 (c) 2020 Georg Thieme Verlag KG Stuttgart . New York
DOI https://doi.org/

10.1055/s-0039-3400260. ISSN 0720-9355. 


\section{Introduction}

Von Willebrand disease (VWD) is the most prevalent inherited bleeding disorder with an autosomal inheritance pattern. It is caused by quantitative and/or functional deficits of von Willebrand factor (VWF), a large multimeric plasma glycoprotein that plays critical roles in hemostasis. VWF mediates platelet adhesion and aggregation in primary hemostasis. ${ }^{1,2}$ Furthermore, VWF carries factor VIII (FVIII) in the circulation, protecting it from rapid proteolytic degradation and delivering it to sites of vascular damage for secondary hemostasis. ${ }^{1}$

The estimated prevalence of VWD ranges from 0.1 to $1 \%$, but only approximately 1 in 10,000 individuals has clinically significant bleeding symptoms. ${ }^{2}$ The diagnosis of VWD is based on a personal history of bleeding and laboratory tests assessing abnormalities in VWF including measurement of VWF antigen levels and assays quantifying the binding activity of VWF to platelet GPIb $\alpha$ and collagen, as well as the coagulant activity of FVIII assay. ${ }^{1}$ Diagnosis and classification of VWD can be challenging because of considerable heterogeneity in its molecular basis and variability in plasma VWF levels and its clinical manifestations. The mean plasma level of VWF is $100 \mathrm{IU} \mathrm{dL}^{-1}$, but the population distribution is between 50 and $200 \mathrm{IU} \mathrm{dL}^{-1}$. Many factors, such as ABO blood group, age, illness, pregnancy, and medication, have an impact on VWF levels, making the diagnosis of VWD difficult. ${ }^{3,4}$ Replacement with plasma-derived VWF concentrates and stimulating the release of VWF from intracellular storages by desmopressin administration are the main therapeutic options for VWD. ${ }^{5}$ However, the effectiveness of the latter treatment is variable between patients and is dependent on the type of genetic defect in VWF. It is expected that most type 1 VWD patients (except patients with accelerated VWF clearance) respond well to desmopressin, whereas type 2 VWD (due to dysfunctional VWF) and type 3 VWD (due to the absence of VWF) do not respond to desmopressin stimulation. ${ }^{5}$ In particular, desmopressin administration is contraindicated in gain-of-function type 2B VWD due to the occurrence of severe thrombocytopenia and a subsequent deficiency of plasma VWF. ${ }^{6}$

In the last three decades, various studies including mutation screening of populations with different ethnic origins, functional analysis of the individual sequence variations in heterologous systems, and genome-wide association studies have made significant progress in our understanding of the genetic etiology and pathogenesis of the VWD. In this review, we will discuss in detail the latest findings on the genetic basis and molecular mechanisms contributing to quantitative VWD types 1 and 3.

\section{Von Willebrand Factor Gene and Protein Structure}

The VWF gene (VWF) is located on the short arm of chromosome 12 (12p13.31), and it spans approximately $178 \mathrm{~kb}$ of genomic DNA. The VWF comprises 52 exons ranging from 40 to $342 \mathrm{bp}$, but exon 28 is exceptionally larger ( $1.4 \mathrm{~kb}$ in size $)^{7}$ The 9-kb VWF transcript encodes a pre-pro-VWF protein of 2,813 amino acids (aa). ${ }^{8}$ The pre-pro-VWF precursor has a mosaic architecture with the following domain structure: D1-D2-D '-D3-A1-A2-A3-D4-C1-C2-C3-C4-C5-C6-CK. ${ }^{7,8}$ The recent studies demonstrated that $\mathrm{D}$ domains each contain a VW domain, a C8 fold, a trypsin inhibitor-like (TIL) structure, and an E module. However, D' lacks the VW domain and C8 fold; the D4 domain lacks the $\mathrm{E}$ module and encompasses distinctive subdomain D4N. ${ }^{8}$

The domains might be characterized as structural or functional, depending on their role in VWF structure or its interaction with other proteins. Structural domains are involved in the posttranslational processing of VWF, for example, C-terminal domains are required for dimerization of VWF monomers and the D1, D2, and D3 domains for multimerization of VWF dimers. $^{9,10}$ The latter domains contain a CGLC consensus sequence that is highly homologous to the active site of protein disulfide isomerase (PDI) which promotes multimerization through interchain disulfide bonding. ${ }^{11}$

Functional domains comprise those that enclose cleavage sites for proteolysis (domain A2) and binding sites for collagen (domains A1 and A3), platelets (domain A1 and C1 for platelet glycoprotein Ib [GPIb] and integrin GPIIb/IIIa receptors, respectively), and FVIII (domains D3 and $\left.\mathrm{D}^{\prime}\right) .^{12-15}$

\section{VWF Biosynthesis}

VWF is produced by endothelial cells and by the platelet precursor, megakaryocytes, accounting for approximately 85 and $15 \%$ of the total VWF present in normal platelet-rich plasma, respectively. ${ }^{16,17}$ The primary VWF product (pre-pro-VWF) undergoes systematic posttranslational modifications including dimerization, glycosylation, removal of propeptide domains (D1 and D2 domains), and eventual formation of high molecular-weight multimers containing up to 100 monomers. ${ }^{18,19}$

In the endoplasmic reticulum (ER), pre-pro-VWF dimerizes "tail-to-tail" over three disulfide bonds formed between the cysteine knot (CK) domains, catalyzed by a family member of PDI (PDIA1). ${ }^{20,21}$ Subsequently, VWF dimers are moved to the Golgi complex, where multimerization and the proteolytic removal of the VWF propeptide occur. Multimerization occurs through additional interchain disulfide bridging between D3 domains. $^{11,19,22}$ Propeptide and D' domain support proper alignment of the pro-VWF dimers, which is crucial for the multimerization process. ${ }^{23}$ Along with the multimer elongation, VWF is packed in storage organelles, which are named Weibel-Palade bodies (WPBs) in endothelial cells and $\alpha$-granules in platelets. ${ }^{24}$ The VWF is secreted from endothelial cells through either a constitutive or a regulated pathway, whereas VWF stored in $\alpha$-granules will be released upon platelet activation. ${ }^{25,26}$

The VWF is extensively glycosylated with $17 \mathrm{~N}$-linked and 10 O-linked oligosaccharide chains, which make up approximately $20 \%$ of the mass of the protein. ${ }^{27,28}$

\section{VWD Classification}

VWD is classified as quantitative types 1 and 3 , and qualitative type 2. Type $1 \mathrm{VWD}$, which is due to a mild to moderate reduction in functionally normal VWF, is the most prevalent of 
the disease constituting 65 to $80 \%$ of VWD cases. ${ }^{4}$ Type 3 is characterized by the virtually complete absence of VWF, and it affects 0.1 to 5.3 per million people depending upon the rate of consanguinity. ${ }^{29}$ Type 2 disease, characterized by functionally abnormal VWF, occurs in 20 to $35 \%$ of patients and is further classified into the four types: $2 \mathrm{~A}, 2 \mathrm{~B}, 2 \mathrm{M}$, and $2 \mathrm{~N}$. The functional defects include enhanced (2B) or loss of $(2 \mathrm{~A}$ and $2 \mathrm{M})$ affinity to platelet receptors or impaired binding to $\mathrm{FVIII}(2 \mathrm{~N}){ }^{4}$

\section{VWD Genetic Basis}

A review of the current mutation databases indicates that genetic etiology and molecular pathogenesis of VWD are extremely heterogeneous. The VWD mutation listings can be found in the online Human Gene Mutation Database (HGMD) (http://www.hgmd.cf.ac.uk/ac/gene.php?gene=VWF) and the Coagulation Factor Variant Databases portal supported by the European Association for Haemophilia and Allied Disorders (EAHAD) (https://grenada.lumc.nl/LOVD2/VWF/home.php? select_db=VWF). In HGMD, 1,027 VWD mutations encompassing 673 missense/nonsense substitutions, 110 splicing variations, 20 regulatory substitutions, 136 small deletions/ insertions/indels, 50 large deletions, 9 large insertions/duplications, 28 complex rearrangements, and 1 repeat variation are recorded (http://www.hgmd.cf.ac.uk/ac/gene.php?gene= VWF; accessed in March 2019).

The genetic investigations have provided extensive insights into genetic etiology of type 2 and type 3 VWD. Pathologic variants have been identified in more than $85 \%$ of type 2 and type 3 VWD populations. ${ }^{29-31}$ In contrast, the genetic pathology of type 1 disease remains incompletely defined in many cases, and pathogenic sequence variations are found in only approximately two-thirds of type $1 \mathrm{VWD}$ patients. Likely, other genetic determinants outside of VWF are involved. ${ }^{30,32-34}$

Although previous studies have largely elucidated the genetic basis of VWD, they may have missed mutations associated with VWD by conventional polymerase change reaction and Sanger DNA sequencing. For instance, these techniques are incompetent to detect large heterozygous deletions (or duplications) because of the presence of the normal VWF allele which can mask the failure in amplification of the deleted allele. Many previous reports, missing methods analyzing gene dosage such as multiplex ligationdependent probe amplification (MLPA), have failed to identify large deletions (or duplications) in heterozygous type 1 and 2 and in compound heterozygous type 3 patients. It is therefore expected that the contribution of copy number variations in the pathogenesis of VWD is underestimated. Furthermore, the former cohort studies have mainly focused on the sequencing of only coding exons of $V W F$ and the immediate surrounding intronic region, and then potential causative mutations in the promoter region (particularly in mild-moderate type $1 \mathrm{VWD}$ ) and deep intronic region are overlooked. Additionally, the synonymous sequence alterations affecting splicing might have been passed over, since their potential impact had been misinterpreted in the first place. In some instances, the deleterious impact of the previously overlooked VWF silent mutations was highlighted later following further RNA transcript studies and reanalysis of genomic DNA. ${ }^{35,36}$

\section{Pathogenic Mechanisms Underlying Type 1 VWD}

Type $1 \mathrm{VWD}$, which is defined by a quantitative deficiency of VWF with plasma VWF levels ranging from 5 to $50 \mathrm{IU} / \mathrm{dL}$, has complex pathogenesis and demonstrates variability in phenotypic penetrance and expressivity. ${ }^{30,32,33}$

Type 1 VWD is a multifactorial trait in which more than one gene and various environmental factors influence plasma levels of $\mathrm{VWF}^{37}$ It is now confirmed that the $\mathrm{ABO}$ blood group is the most imperative genetic modifier of plasma VWF levels, such that plasma VWF levels in individuals with blood group 0 are approximately $25 \%$ lower compared with those in non-O blood group individuals. ${ }^{37}$ This is likely due to glycan structures presented on group O-VWF, which lead to accelerated clearance of the protein. ${ }^{38}$ Coinheritance of blood group $O$ has an important pathogenic role in type 1 VWD. It is demonstrated that blood group $\mathrm{O}$ is overrepresented in type $1 \mathrm{VWD}$ subjects (73\% of type $1 \mathrm{VWD}$ cases vs. $45 \%$ of the general population). ${ }^{32,39}$

Over the last decade, several groups have studied the genetic basis of type $1 \mathrm{VWD}$ cohorts of the European and North American populations. Remarkably, the results from all of these studies have steadily reported that VWF candidate mutations have been identified in approximately $65 \%$ of index cases. ${ }^{30,32-34}$ Furthermore, data from these studies indicated that the likelihood of detecting a VWF mutation rises with lower VWF levels (VWF:Ag $<30 \mathrm{IU} / \mathrm{dL}$ ). The large multicenter studies of type $1 \mathrm{VWD}$ cases from the United States (Zimmerman program), European (MCMDM-1 VWD study), and Canadian cohorts reported that overall approximately $82 \%$ of cases had VWF mutations when the VWF:Ag was $\leq 30 \mathrm{IU} / \mathrm{dL}$, whereas only approximately $50 \%$ of cases with VWF:Ag $>30 \mathrm{IU} / \mathrm{dL}$ had VWF mutations. ${ }^{32-34}$

\section{Genetic Variants within VWF}

To date, more than 250 different candidate mutations have been documented in HGMD for type 1 VWD which comprise candidate missense variations (accounting for the majority of pathogenic variants: $\sim 64 \%$ ), splice-site variations $(\sim 15 \%)$, promoter sequence variations ( $\sim 5 \%)$, small deletions ( $\sim 5 \%)$, small insertions $(\sim 3 \%)$, nonsense mutations $(\sim 4 \%)$, gene conversions ( $2 \%$ ), and large deletions ( 2\%) (http://www.hgmd.cf.ac.uk/ ac/gene.php?gene=VWF, accessed in March 2019; -Fig. 1A). Although type $1 \mathrm{VWD}$ is mainly due to only one affected allele and inherited in an autosomal-dominant manner, approximately $15 \%$ of patients have more than one candidate mutation (homozygous and compound heterozygous). ${ }^{33}$ Notably, some assigned type 1 VWD mutations were later considered to be reclassified as VWD type 2 ( $2 \mathrm{~A}$ or $2 \mathrm{M}$ ) due to displaying subtle abnormal multimers following completion of the MCMDM-1 VWD study ${ }^{40}$ In - Table 1, the mutations listed in databases for both type 1 and type 2 VWD are marked by an asterisk. Furthermore, in some patients, VWD type 1 is caused by 

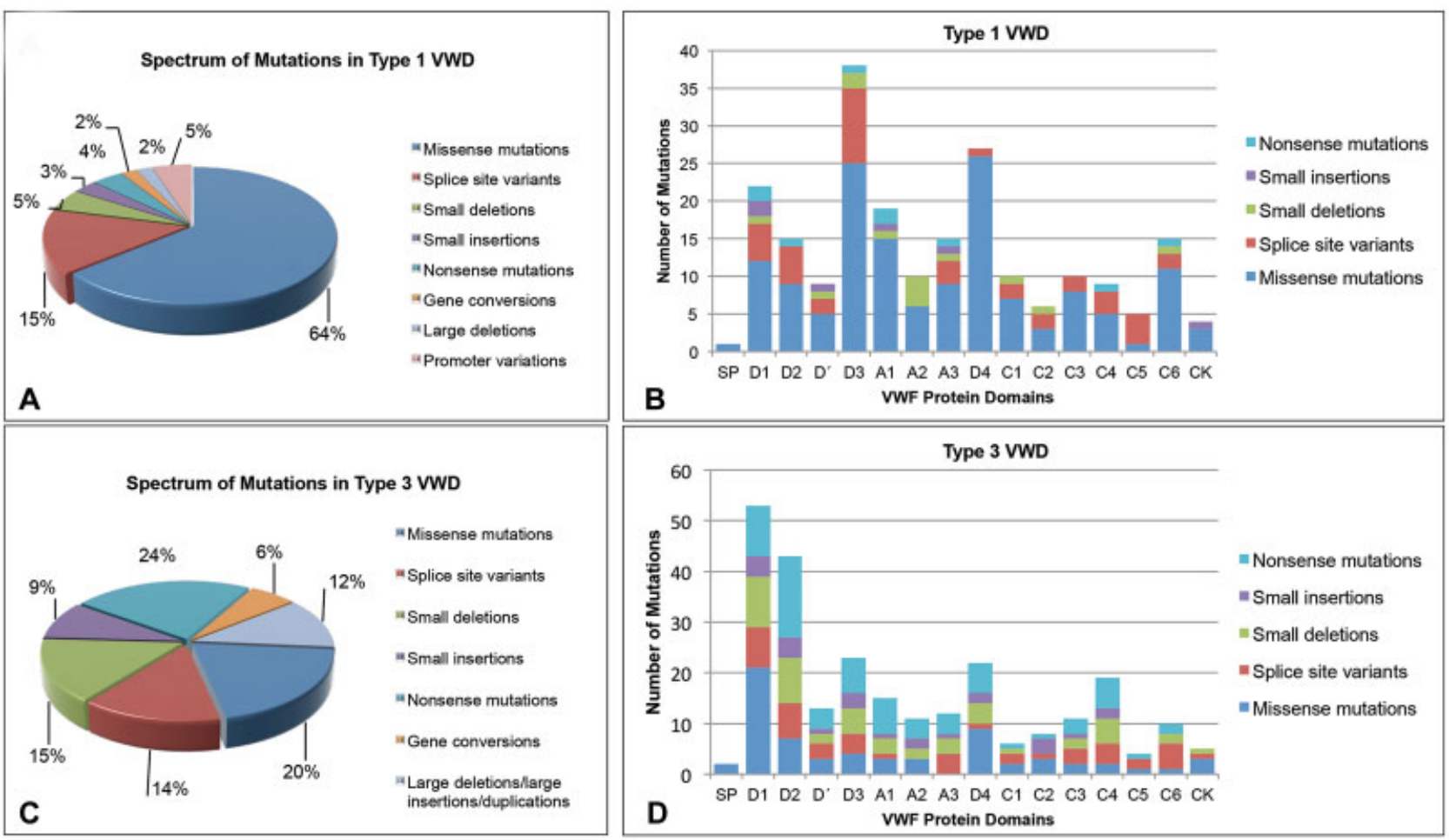

Fig. 1 Spectrum and distribution of mutations in type 1 and type 3 VWD. Panels (A) and (C) demonstrate spectrum and frequency (\%) of mutations in type 1 and type 3 VWD, respectively. As it is shown, in type 1 VWD missense mutations are predominant (64\%), while in type 3 VWD null alleles (splice site variants, small deletions/insertions, nonsense mutations, large deletions, large insertions, duplications, and gene conversions) account for the majority of the mutations. Panels (B) and (D) show the distribution of mutations on VWF domains in type 1 and type 3 VWD, respectively. VWD, von Willebrand disease.

dominant-negative mutations, such as p.Cys1130Phe and p. Cys1149Arg. Heterodimerization of variant and normal VWF subunits leads to intracellular retention of both variant and normal VWF and consequently results in a dominant-negative effect on the secretion of $\mathrm{VWF}^{4,41,42}$ Until now, the pathogenic mechanisms causing quantitative type $1 \mathrm{VWD}$, in general, include decreased VWF synthesis, impaired VWF storage and secretion, and accelerated VWF clearance (-Fig. 2). The decreased VWF synthesis might be due to promoter mutations disrupting binding of transcription factors as well as null mutations (nonsense, deletion, and splice-site variations) resulting in no stable VWF mRNA production or truncated proteins retained in the ER. In the HGMD registry, several sequence variations in the promoter region of the VWF are recorded for type $1 \mathrm{VWD}$, but the pathogenicity of these variations has not been yet verified. More than 30 putative splicing mutations are reported for type 1 VWD of which some are confirmed to be leading to aberrantly spliced VWF transcripts. The RNA transcript studies done by several groups showed that these splicing variants might lead to either exon skipping, cryptic splice-site activation, or intron retention, thus resulting in truncated proteins. ${ }^{35,43-46}$ An interesting consensus splice-site mutation is c.1534-3C $>$ A, because it is identified as both homozygous and heterozygous in type $1 \mathrm{VWD}$ cases. This splice-site mutation impairs but does not abolish normal mRNA splicing. It simultaneously induces skipping of the exon 14, activation of a cryptic splice site, and a normal VWF transcription. Interestingly, the cases which are heterozygous for the c.1534-3C > A mutation always had low platelet VWF levels, but sometimes circulating VWF levels within the normal range. ${ }^{43}$ This indicates the possibility of a discrepant splicing procedure in endothelial cells and platelet precursors, megakaryocytes, which should be taken into consideration in future studies.

Missense variations in type 1 VWD either impair VWF storage and secretion or cause accelerated VWF clearance from the plasma circulation ( - Table 1; - Fig. 2). The missense mutations affecting storage and secretion of the VWF are spread throughout all VWF domains and the molecular mechanisms by which these mutations cause defective intracellular packaging and secretion of VWF are not completely characterized ( - Fig. 1B). It is believed that some of these mutations cause substantial structural changes in VWF protein, such that the ER quality control system prevents trafficking to the Golgi compartment. The retained VWF protein either aggregates in the ER or undergoes intracellular degradation that eventually leads to less WPB storage and less production of $\mathrm{VWF}^{47-56}$ ( - Table 1). Wang et al suggested that all cysteine mutations involved in intrachain disulfide bonds (e.g., p.Cys1060Tyr, p.Cys1130Phe, and p.Cys1149Arg) may disrupt the conformation of VWF, causing ER retention. ${ }^{54}$ Alternatively, some of the mutations (with mild ER retention or only dilated ER) mainly disturb tubule formation and normal storage of VWF, leading to diminished exocytosis of WPBs and consequently resulting in less VWF secretion (e.g., p.Cys2190Tyr, p.Ala1716Pro, and p. Tyr1584Cys; - Table 1). $48,50,55,57$ 
Table 1 List of missense mutations detected in type 1 VWD and their impact on VWF biosynthesis based on in vitro and in vivo functional studies

\begin{tabular}{|c|c|c|}
\hline aa Exchange & Domain & $\begin{array}{l}\text { Functional characterization/ } \\
\text { impact on splicing }\end{array}$ \\
\hline p.Gly19Arg 33 & SP & $\begin{array}{l}\text { No impact on the expression of } \\
\text { VWF/Putative impact on } \\
\text { splicing }{ }^{49}\end{array}$ \\
\hline p.Ser49Arg ${ }^{107}$ & D1/VWD & $\mathrm{n} / \mathrm{a}$ \\
\hline p.Leu60Pro ${ }^{108}$ & D1/VWD & $\mathrm{n} / \mathrm{a}$ \\
\hline p.Asn99Ser ${ }^{31}$ & D1/VWD & $\mathrm{n} / \mathrm{a}$ \\
\hline p.Leu129Met ${ }^{34}$ & D1/VWD & $\mathrm{n} / \mathrm{a}$ \\
\hline p.Ala138Asp ${ }^{31}$ & D1/VWD & $\mathrm{n} / \mathrm{a}$ \\
\hline p.Asp141Gly 50 & D1/VWD & $\begin{array}{l}\text { Intracellular retention; impaired } \\
\text { secretion } 49\end{array}$ \\
\hline p.Gly160Trp 33 & D1/VWD & $\begin{array}{l}\text { Intracellular retention; impaired } \\
\text { secretion } 49\end{array}$ \\
\hline p.Cys162Arg ${ }^{109}$ & D1/VWD & $\mathrm{n} / \mathrm{a}$ \\
\hline p.Asn $1661 \mathrm{e}^{33}$ & D1/VWD & $\mathrm{n} / \mathrm{a}$ \\
\hline p.Cys237Trp ${ }^{109}$ & $\mathrm{D} 1 / \mathrm{C} 8$ & $\mathrm{n} / \mathrm{a}$ \\
\hline p.Arg324Pro ${ }^{107}$ & $\mathrm{D} 1 / \mathrm{TIL}$ & $\mathrm{n} / \mathrm{a}$ \\
\hline p.Cys325Phe ${ }^{31}$ & $\mathrm{D} 1 / \mathrm{TIL}$ & $\mathrm{n} / \mathrm{a}$ \\
\hline p.Arg491Pro ${ }^{101}$ & $\mathrm{D} 2 / \mathrm{C} 8$ & $\mathrm{n} / \mathrm{a}$ \\
\hline p.Asp558Tyr ${ }^{88}$ & $\mathrm{D} 2 / \mathrm{C} 8$ & $\mathrm{n} / \mathrm{a}$ \\
\hline p.Met576Ile & $\mathrm{D} 2 / \mathrm{C} 8$ & $\mathrm{n} / \mathrm{a}$ \\
\hline p.Ala594Gly ${ }^{110}$ & $\mathrm{D} 2 / \mathrm{C} 8$ & $\mathrm{n} / \mathrm{a}$ \\
\hline p.Ala641Val ${ }^{33}$ & $\mathrm{D} 2 / \mathrm{C} 8$ & $\mathrm{n} / \mathrm{a}$ \\
\hline p.Cys707Arg ${ }^{101}$ & $\mathrm{D} 2 / \mathrm{TIL}$ & $\mathrm{n} / \mathrm{a}$ \\
\hline p.Met736Thr ${ }^{31}$ & $\mathrm{D} 2 / \mathrm{E}$ & $\mathrm{n} / \mathrm{a}$ \\
\hline p.Met740lle 42 & $\mathrm{D} 2 / \mathrm{E}$ & $\mathrm{n} / \mathrm{a}$ \\
\hline p.Lys762Glu ${ }^{34}$ & $\mathrm{D} 2 / \mathrm{E}$ & $\mathrm{n} / \mathrm{a}$ \\
\hline p.Arg768Gln 60 & $\mathrm{D}^{\prime} / \mathrm{TIL}$ & $\mathrm{n} / \mathrm{a}$ \\
\hline p.Met771lle 33 & $\mathrm{D}^{\prime} / \mathrm{TIL}$ & $\begin{array}{l}\text { A combination of retention in ER } \\
\text { along with impaired storage in } \\
\text { WPBs and secretion }\end{array}$ \\
\hline p.Met771Val ${ }^{56}$ & $\mathrm{D}^{\prime} / \mathrm{TIL}$ & $\begin{array}{l}\text { A combination of ER retention } \\
\text { along with impaired storage, } \\
\text { multimerization, and secretion }\end{array}$ \\
\hline p.Arg782Gln ${ }^{56}$ & $\mathrm{D}^{\prime} / \mathrm{TIL}$ & $\begin{array}{l}\text { A combination of retention in ER } \\
\text { along with impaired storage in } \\
\text { WPBs and secretion } \\
56\end{array}$ \\
\hline p.Asn857Ser ${ }^{30}$ & $\overline{D^{\prime} / E}$ & $\mathrm{n} / \mathrm{a}$ \\
\hline p.Leu881Arg ${ }^{33}$ & D3/VWD & $\begin{array}{l}\text { Decreased secretion (only in } \\
\text { single expression but not for } \\
\text { co-expression), normal storage }\end{array}$ \\
\hline p.Arg924Trp ${ }^{34}$ & D3/VWD & $\begin{array}{l}\text { No impact on expression and } \\
\text { function of VWF }\end{array}$ \\
\hline p.Phe1021Cys ${ }^{31}$ & D3/VWD & $\mathrm{n} / \mathrm{a}$ \\
\hline p.Cys1060Tyr ${ }^{36}$ & D3/VWD & $\mathrm{n} / \mathrm{a}$ \\
\hline p.Ala1108Pro ${ }^{109}$ & D3/VWD & $\mathrm{n} / \mathrm{a}$ \\
\hline p.Cys $1111 \mathrm{Tyr}^{34}$ & $\mathrm{D} 3 / \mathrm{C} 8$ & $\mathrm{n} / \mathrm{a}$ \\
\hline p.Cys $1130 \mathrm{Phe}^{* 40}$ & $\mathrm{D} 3 / \mathrm{TIL}$ & $\begin{array}{l}\text { Associated with accelerated VWF } \\
\text { clearance }^{64} \text {; ER retention, } \\
\text { reduced storage, and secretion }\end{array}$ \\
\hline
\end{tabular}

(Continued)
Table 1 (Continued)

\begin{tabular}{|c|c|c|}
\hline aa Exchange & Domain & $\begin{array}{l}\text { Functional characterization/ } \\
\text { impact on splicing }\end{array}$ \\
\hline p.Cys1130Gly*40 & $\mathrm{D} 3 / \mathrm{TIL}$ & $\begin{array}{l}\text { Associated with accelerated VWF } \\
\text { clearance }^{64} \text {; intracellular } \\
\text { retention, impaired secretion }{ }^{51}\end{array}$ \\
\hline p.Cys1130Arg ${ }^{* 40}$ & $\mathrm{D} 3 / \mathrm{TIL}$ & $\begin{array}{l}\text { Associated with accelerated VWF } \\
\text { clearance }^{64} \text {; intracellular } \\
\text { retention, impaired secretion }\end{array}$ \\
\hline p.Trp1144Gly ${ }^{59}$ & $\mathrm{D} 3 / \mathrm{TIL}$ & $\begin{array}{l}\text { Associated with accelerated VWF } \\
\text { clearance }^{59} \text {; intracellular } \\
\text { retention, impaired secretion }\end{array}$ \\
\hline p.Arg1145Cys ${ }^{111}$ & $\mathrm{D} 3 / \mathrm{TIL}$ & $\mathrm{n} / \mathrm{a}$ \\
\hline p.Tyr1146Cys ${ }^{34}$ & $\mathrm{D} 3 / \mathrm{TIL}$ & $\begin{array}{l}\text { Impaired pseudo-WPBs forma- } \\
\text { tion and secretion, reduction in } \\
\text { large multimers }\end{array}$ \\
\hline p.Cys1149Arg*53 & $\mathrm{D} 3 / \mathrm{TIL}$ & $\begin{array}{l}\text { Associated with accelerated VWF } \\
\text { clearance } 59 \text {; ER retention, } \\
\text { impaired storage and secretion } \\
\end{array}$ \\
\hline p.Thr1156Met ${ }^{56}$ & $\mathrm{D} 3 / \mathrm{TIL}$ & $\begin{array}{l}\text { ER retention, impaired pseudo- } \\
\text { WPB formation and secretion, } \\
\text { loss of large multimers }{ }^{56}\end{array}$ \\
\hline p.Glu1161 Lys ${ }^{31}$ & D3/TIL & $\mathrm{n} / \mathrm{a}$ \\
\hline p.Cys1165Phe ${ }^{103}$ & $\mathrm{D} 3 / \mathrm{TIL}$ & $\mathrm{n} / \mathrm{a}$ \\
\hline p.Cys1190Arg 34 & $\mathrm{D} 3 / \mathrm{TIL}$ & $\mathrm{n} / \mathrm{a}$ \\
\hline p.Arg1205Cys ${ }^{62}$ & $\mathrm{D} 3 / \mathrm{E}$ & $\begin{array}{l}\text { Associated with accelerated VWF } \\
\text { clearance }{ }^{60} \text {; macrophage-medi- } \\
\text { ated clearance of VWF in vivo }\end{array}$ \\
\hline p.Arg1205Ser ${ }^{62}$ & D3/E & $\begin{array}{l}\text { Associated with accelerated VWF } \\
\text { clearance }{ }^{60} \text {; macrophage-medi- } \\
\text { ated clearance of VWF in vivo }{ }^{62}\end{array}$ \\
\hline p.Arg1205His ${ }^{62}$ & $\mathrm{D} 3 / \mathrm{E}$ & $\begin{array}{l}\text { Associated with accelerated VWF } \\
\text { clearance }{ }^{60} \text {; macrophage-medi- } \\
\text { ated clearance of VWF in vivo }{ }^{62}\end{array}$ \\
\hline p.Cys1227Arg*112 & $\mathrm{D} 3 / \mathrm{E}$ & $\mathrm{n} / \mathrm{a}$ \\
\hline p.Val1229Gly ${ }^{34}$ & $\mathrm{D} 3 / \mathrm{E}$ & $\mathrm{n} / \mathrm{a}$ \\
\hline p.Asn1231Thr ${ }^{34}$ & $\mathrm{D} 3 / \mathrm{E}$ & $\mathrm{n} / \mathrm{a}$ \\
\hline p.Asn1231His ${ }^{31}$ & $\bar{D} 3 / \mathrm{E}$ & $\mathrm{n} / \mathrm{a}$ \\
\hline p.Pro1266Leu ${ }^{113}$ & $\bar{D} 3 / \mathrm{E}$ & $\mathrm{n} / \mathrm{a}$ \\
\hline p.Val1279lle ${ }^{* 2}$ & $\mathrm{~A} 1$ & $\mathrm{n} / \mathrm{a}$ \\
\hline p.Ser1285Pro ${ }^{33}$ & $\mathrm{~A} 1$ & $\begin{array}{l}\text { ER retention, shorter and } \\
\text { rounder WPBs, and impaired } \\
\text { secretion }\end{array}$ \\
\hline p.Leu1307Pro*40 & $\mathrm{A} 1$ & $\begin{array}{l}\text { ER retention, less number of } \\
\text { WPBs, shorter and rounder } \\
\text { WPBs, impaired secretion }{ }^{50}\end{array}$ \\
\hline p.Arg1315Cys*40 & $\mathrm{A} 1$ & $\begin{array}{l}\text { Associated with accelerated VWF } \\
\text { clearance }^{58}\end{array}$ \\
\hline p.A1 g1315His ${ }^{40}$ & A1 & $\mathrm{n} / \mathrm{a}$ \\
\hline p.Arg1315Leu*40 & A1 & $\mathrm{n} / \mathrm{a}$ \\
\hline p.Arg1342Cys ${ }^{33}$ & $\mathrm{~A} 1$ & $\mathrm{n} / \mathrm{a}$ \\
\hline p.Leu1361Ser 33 & $\mathrm{~A} 1$ & $\begin{array}{l}\text { Associated with accelerated VWF } \\
\text { clearance } 58\end{array}$ \\
\hline p.Arg1374Cys"58 & $\mathrm{A} 1$ & $\begin{array}{l}\text { Associated with accelerated VWF } \\
\text { clearance }^{58}\end{array}$ \\
\hline p.Ser1378Phe ${ }^{* 40}$ & $\mathrm{~A} 1$ & $\mathrm{n} / \mathrm{a}$ \\
\hline p.Arg1379Cys ${ }^{112}$ & A1 & $\mathrm{n} / \mathrm{a}$ \\
\hline p.Pro1413Leu ${ }^{33}$ & $\mathrm{~A} 1$ & $\mathrm{n} / \mathrm{a}$ \\
\hline
\end{tabular}

(Continued) 
Table 1 (Continued)

\begin{tabular}{|c|c|c|}
\hline aa Exchange & Domain & $\begin{array}{l}\text { Functional characterization/ } \\
\text { impact on splicing }\end{array}$ \\
\hline p.Gly 1415 Asp $^{33}$ & $\mathrm{~A} 1$ & $\begin{array}{l}\text { Associated with accelerated VWF } \\
\text { clearance }^{58}\end{array}$ \\
\hline p.lle1416Asn $* 40$ & $\mathrm{~A} 1$ & $\begin{array}{l}\text { Associated with accelerated VWF } \\
\text { clearance }^{60}\end{array}$ \\
\hline p.Glu1447Gln ${ }^{114}$ & $\mathrm{~A} 1$ & $\mathrm{n} / \mathrm{a}$ \\
\hline p.Arg1564Trp ${ }^{31}$ & $\mathrm{~A} 2$ & $\mathrm{n} / \mathrm{a}$ \\
\hline p.Arg1583Trp 33 & $\mathrm{~A} 2$ & $\begin{array}{l}\text { Not pathologic: normal WPBs, } \\
\text { normal secretion }{ }^{50}\end{array}$ \\
\hline p.Tyr1584Cys ${ }^{50}$ & $\mathrm{~A} 2$ & $\begin{array}{l}\text { Normal secretion }{ }^{50} \text {; increased } \\
\text { susceptibility to ADAMTS13 }\end{array}$ \\
\hline p.Lys1617Arg ${ }^{109}$ & $\mathrm{~A} 2$ & $\mathrm{n} / \mathrm{a}$ \\
\hline p.asn1635Thr ${ }^{114}$ & $\mathrm{~A} 2$ & $\mathrm{n} / \mathrm{a}$ \\
\hline p.Arg1668Ser ${ }^{34}$ & $\mathrm{~A} 2$ & $\mathrm{n} / \mathrm{a}$ \\
\hline p.Ala1716Pro ${ }^{48}$ & A3 & $\begin{array}{l}\text { A combination of ER retention } \\
\text { along with impaired storage and } \\
\text { secretion }{ }^{48}\end{array}$ \\
\hline p.Val1758Ala 109 & A3 & $\mathrm{n} / \mathrm{a}$ \\
\hline p.Val1760lle & $\mathrm{A} 3$ & $\begin{array}{l}\text { Associated with accelerated VWF } \\
\text { clearance }^{58}\end{array}$ \\
\hline p.Leu1774Ser ${ }^{33}$ & A3 & $\mathrm{n} / \mathrm{a}$ \\
\hline p.Arg1779Leu ${ }^{31}$ & $\mathrm{~A} 3$ & $\mathrm{n} / \mathrm{a}$ \\
\hline p.Lys1794Glu ${ }^{33}$ & $\mathrm{~A} 3$ & $\begin{array}{l}\text { Associated with accelerated VWF } \\
\text { clearance }^{58}\end{array}$ \\
\hline p.Asn1818Ser ${ }^{34}$ & A3 & $\mathrm{n} / \mathrm{a}$ \\
\hline p.Val1822Gly 33 & A3 & $\begin{array}{l}\text { Associated with accelerated VWF } \\
\text { clearance, }{ }^{58} \text { ER retention, } \\
\text { shorter and less number WPBs }{ }^{50}\end{array}$ \\
\hline p.Arg1837Trp ${ }^{115}$ & A3 & $\mathrm{n} / \mathrm{a}$ \\
\hline p.Cys1879Phe ${ }^{115}$ & D4/D4N & $\mathrm{n} / \mathrm{a}$ \\
\hline p.Lys1887Asn ${ }^{115}$ & D4/D4N & $\mathrm{n} / \mathrm{a}$ \\
\hline p.Cys1899Arg ${ }^{101}$ & D4/D4N & $\mathrm{n} / \mathrm{a}$ \\
\hline p.His1900Pro ${ }^{31}$ & D4/D4N & $\mathrm{n} / \mathrm{a}$ \\
\hline p.Pro1933Leu ${ }^{115}$ & D4/D4N & $\mathrm{n} / \mathrm{a}$ \\
\hline p.Glu1938Asp ${ }^{115}$ & D4/D4N & $\mathrm{n} / \mathrm{a}$ \\
\hline p.Thr1951Ala ${ }^{115}$ & D4/VWD & $\mathrm{n} / \mathrm{a}$ \\
\hline p.Pro2063Ser ${ }^{34}$ & D4/VWD & $\begin{array}{l}\text { No impact on in vitro VWF } \\
\text { expression }^{49}\end{array}$ \\
\hline p.Gly2083Asp ${ }^{101}$ & D4/VWD & $\mathrm{n} / \mathrm{a}$ \\
\hline p.Cys2085Phe ${ }^{101}$ & D4/VWD & $\mathrm{n} / \mathrm{a}$ \\
\hline p.Thr2104lle & D4/VWD & $\mathrm{n} / \mathrm{a}$ \\
\hline p.Pro2145Ser ${ }^{33}$ & $\mathrm{D} 4 / \mathrm{C} 8$ & $\mathrm{n} / \mathrm{a}$ \\
\hline p.Cys2150Tyr ${ }^{31}$ & $\mathrm{D} 4 / \mathrm{C} 8$ & $\mathrm{n} / \mathrm{a}$ \\
\hline p.Val2153Phe 31 & $\mathrm{D} 4 / \mathrm{C} 8$ & $\mathrm{n} / \mathrm{a}$ \\
\hline p.Ser2179Phe ${ }^{4}$ & $\mathrm{D} 4 / \mathrm{C} 8$ & $\begin{array}{l}\text { Associated with accelerated VWF } \\
\text { clearance }{ }^{59}\end{array}$ \\
\hline p.Cys2184Tyr ${ }^{31}$ & $\mathrm{D} 4 / \mathrm{C} 8$ & $\mathrm{n} / \mathrm{a}$ \\
\hline p.Arg2185Trp 34 & $\mathrm{D} 4 / \mathrm{C} 8$ & $\mathrm{n} / \mathrm{a}$ \\
\hline p.Arg2185Gln 34 & $\mathrm{D} 4 / \mathrm{C} 8$ & $\mathrm{n} / \mathrm{a}$ \\
\hline p.Cys2190Tyr ${ }^{48}$ & $\mathrm{D} 4 / \mathrm{C} 8$ & \\
\hline
\end{tabular}

Table 1 (Continued)

\begin{tabular}{|c|c|c|}
\hline aa Exchange & Domain & $\begin{array}{l}\text { Functional characterization/ } \\
\text { impact on splicing }\end{array}$ \\
\hline & & $\begin{array}{l}\text { A combination of slightly ER } \\
\text { colocalization along with } \\
\text { drastically impaired storage and } \\
\text { secretion }^{48}\end{array}$ \\
\hline p.Cys2190Trp 31 & $\mathrm{D} 4 / \mathrm{C} 8$ & $\mathrm{n} / \mathrm{a}$ \\
\hline p.Leu2207Pro ${ }^{33}$ & $\mathrm{D} 4 / \mathrm{TIL}$ & $\begin{array}{l}\text { Intracellular retention, impaired } \\
\text { secretion } 49\end{array}$ \\
\hline p.Glu2233Gly 34 & $\mathrm{D} 4 / \mathrm{TIL}$ & $\mathrm{n} / \mathrm{a}$ \\
\hline p.Glu2233Lys ${ }^{31}$ & $\mathrm{D} 4 / \mathrm{TIL}$ & $\mathrm{n} / \mathrm{a}$ \\
\hline p.Glu2233Val ${ }^{31}$ & $\mathrm{D} 4 / \mathrm{TIL}$ & $\mathrm{n} / \mathrm{a}$ \\
\hline p.Cys2237Phe ${ }^{108}$ & D4/TIL & $\mathrm{n} / \mathrm{a}$ \\
\hline p.Cys2254Tyr ${ }^{31}$ & $\mathrm{D} 4 / \mathrm{TIL}$ & $\mathrm{n} / \mathrm{a}$ \\
\hline p.Cys2257Ser 33 & $\mathrm{C} 1$ & $\begin{array}{l}\text { Intracellular retention, impaired } \\
\text { secretion }{ }^{49}\end{array}$ \\
\hline p.Arg2287Trp ${ }^{116}$ & $\mathrm{C} 1$ & $\begin{array}{l}\text { Pathologic mild reduction in } \\
\text { secretion } 49\end{array}$ \\
\hline p.Asn2290Tyr ${ }^{5}$ & $\mathrm{C} 1$ & $\mathrm{n} / \mathrm{a}$ \\
\hline p.Cys2304Tyr 33 & $\mathrm{C} 1$ & $\begin{array}{l}\text { Marked intracellular retention, } \\
\text { impaired secretion } 49\end{array}$ \\
\hline p.Arg2311His ${ }^{110}$ & $\mathrm{C} 1$ & $\mathrm{n} / \mathrm{a}$ \\
\hline p.Arg2313His 33 & C1 & $\mathrm{n} / \mathrm{a}$ \\
\hline p.Cys 2327 Tyr $^{115}$ & $\mathrm{C} 1$ & $\mathrm{n} / \mathrm{a}$ \\
\hline p.Cys $2340 \operatorname{Arg}^{34}$ & $\mathrm{C} 2$ & $\mathrm{n} / \mathrm{a}$ \\
\hline p.Gly2343Val $\left.\right|^{34}$ & $\mathrm{C} 2$ & $\mathrm{n} / \mathrm{a}$ \\
\hline p.Arg2379Cys ${ }^{34}$ & $\mathrm{C2}$ & $\mathrm{n} / \mathrm{a}$ \\
\hline p.Gly2441Cys 33 & $\mathrm{C3}$ & $\begin{array}{l}\text { Associated with accelerated VWF } \\
\text { clearance }^{58} \text {; marked intracellular } \\
\text { retention, impaired secretion } 49\end{array}$ \\
\hline p.Cys2448Tyr ${ }^{115}$ & $\mathrm{C3}$ & $\mathrm{n} / \mathrm{a}$ \\
\hline p.Cas2451Tyr ${ }^{31}$ & C3 & $\mathrm{n} / \mathrm{a}$ \\
\hline p.Arg2464Cys 33 & $\mathrm{C3}$ & $\begin{array}{l}\text { Mild reduction in secretion, } \\
\text { faster-running multimer bands } 49\end{array}$ \\
\hline p.Cys2468Arg ${ }^{31}$ & $\mathrm{C3}$ & $\mathrm{n} / \mathrm{a}$ \\
\hline p.Ser2469Pro ${ }^{33}$ & $\mathrm{C3}$ & $\mathrm{n} / \mathrm{a}$ \\
\hline p.Cys2477Ser ${ }^{33}$ & $\mathrm{C3}$ & $\begin{array}{l}\text { Intracellular retention, impaired } \\
\text { secretion }^{49}\end{array}$ \\
\hline p.Cys2477Tyr ${ }^{33}$ & $\mathrm{C3}$ & $\begin{array}{l}\text { Intracellular retention, impaired } \\
\text { secretion } 49\end{array}$ \\
\hline p.Ser2497Pro 33 & C4 & $\mathrm{n} / \mathrm{a}$ \\
\hline p.Gly2518Ser ${ }^{33}$ & C4 & $\begin{array}{l}\text { Mild reduction in secretion, } \\
\text { faster-running multimer bands, } \\
\text { no impact on VWF clearance } 49\end{array}$ \\
\hline p.Gln2520Pro 33 & C4 & $\begin{array}{l}\text { Associated with accelerated VWF } \\
\text { clearance, }{ }^{59} \text { reduction in secre- } \\
\text { tion, faster-running multimers }{ }^{49}\end{array}$ \\
\hline p.Arg2535Pro ${ }^{31}$ & C4 & $\mathrm{n} / \mathrm{a}$ \\
\hline p.Gly2560Val ${ }^{115}$ & C4 & $\mathrm{n} / \mathrm{a}$ \\
\hline p.Cys2619Tyr ${ }^{30}$ & $\mathrm{C5}$ & $\begin{array}{l}\text { Disrupting intrachain disulfide } \\
\text { bond, intracellular retention, } \\
\text { impaired secretion }\end{array}$ \\
\hline p.Thr2647Met ${ }^{34}$ & C6 & $\mathrm{n} / \mathrm{a}$ \\
\hline p.Lys $2662 \operatorname{Arg}^{29}$ & C6 & $\mathrm{n} / \mathrm{a}$ \\
\hline
\end{tabular}


Table 1 (Continued)

\begin{tabular}{|c|c|c|}
\hline aa Exchange & Domain & $\begin{array}{l}\text { Functional characterization/ } \\
\text { impact on splicing }\end{array}$ \\
\hline p.Arg2663Pro ${ }^{33}$ & C6 & $\mathrm{n} / \mathrm{a}$ \\
\hline p.Arg2663Cys ${ }^{48}$ & C6 & $\begin{array}{l}\text { ER retention, impaired } \\
\text { secretion }{ }^{48}\end{array}$ \\
\hline p.Cys2671Gly ${ }^{31}$ & C6 & $\mathrm{n} / \mathrm{a}$ \\
\hline p.Cys2676Tyr ${ }^{31}$ & $\mathrm{C6}$ & $\mathrm{n} / \mathrm{a}$ \\
\hline p.Cys 2676 Phe $^{30}$ & $\mathrm{C} 6$ & $\begin{array}{l}\text { Disrupting intrachain disulfide } \\
\text { bond, intracellular retention, } \\
\text { impaired secretion }\end{array}$ \\
\hline p.Asn2679Ser ${ }^{31}$ & C6 & $\mathrm{n} / \mathrm{a}$ \\
\hline p.Cys2693Tyr ${ }^{33}$ & C6 & $\begin{array}{l}\text { ER retention, abnormal storage } \\
\text { in WPBs, and impaired } \\
\text { secretion }^{57}\end{array}$ \\
\hline p.Pro2695Arg ${ }^{117}$ & $\mathrm{C6}$ & $\mathrm{n} / \mathrm{a}$ \\
\hline p.Pro2722Ala 33 & C6 & $\mathrm{n} / \mathrm{a}$ \\
\hline p.Pro2776Leu 31 & CK & $\mathrm{n} / \mathrm{a}$ \\
\hline p.Leu2786Pro ${ }^{31}$ & $\overline{C K}$ & $\mathrm{n} / \mathrm{a}$ \\
\hline p.Cys2804Tyr ${ }^{91}$ & CK & $\mathrm{n} / \mathrm{a}$ \\
\hline
\end{tabular}

Abbreviations: $E R$, endoplasmic reticulum; $n / a$ : no answer; TIL, trypsin inhibitor-like; WPBs, Weibel-Palade bodies; VWD, von Willebrand disease; VWF, von Willebrand factor.

Notes: List of type 1 missense mutations published in the literature with references. The location of the mutations on VWF domains and subdomains is based on updated domain designations. ${ }^{8}$ The impact of these missense variations on VWF biosynthesis (if reported) is given with references. The symbol “*” indicates type $1 \mathrm{VWD}$ mutations which are also reported as type 2 (2A and/or 2M) VWD. The gray lines indicate mutations associated with accelerated VWF clearance.
On the other hand, type 1 mutations associated with accelerated VWF clearance, causing reduction in half-life of VWF (to 1-3 hours, compared with 8-12 hours for normal $\mathrm{VWF}$ ), lead to severe reduction in plasma levels of VWF:Ag $(\sim 10-15 \mathrm{IU} / \mathrm{dL})$ in the affected patients. ${ }^{58,59}$ Type $1 \mathrm{VWD}$ patients with increased VWF clearance, which comprise approximately 15 to $20 \%$ of type $1 \mathrm{VWD}$ cases, are characterized by an elevated ratio of VWFpp to VWF:Ag (a ratio of $>2.2$ ) and a rapid elimination of VWF from circulation following desmopressin administration. ${ }^{58-61}$ Using the VWFpp/VWF:Ag ratio, more than 20 different missense substitutions are associated with the elevated clearance phenotype ( - Table 1, lines marked in gray). ${ }^{58,62,63}$ Remarkably, these mutations are not only associated with VWD type 1 but can also be linked to VWD types $2 \mathrm{~A}, 2 \mathrm{~B}$, and $3 .^{58}$ The type 1 mutations provoking accelerated VWF clearance are mostly located in the VWF D3 domain (missense variations p.Arg1205His/Cys/Ser, p.Cys1130Phe/Gly/ Arg, p.Trp1144Gly, and p.Cys1149Arg). ${ }^{31,59,64}$ It is believed that the D3 domain may contain regulatory or recognition sites for VWF survival and clearance. ${ }^{59}$ The half-life of VWF is modulated by several different mechanisms including VWF glycans, shear force exposure which alters the tertiary conformation of the protein as well as receptors expressed on the sinusoidal endothelial cells and on the hepatic and splenic macrophages. ${ }^{65}$ The recent investigations demonstrated that carbohydrate determinants presented on VWF protein modulate VWF clearance through both hepatic asialoglycoprotein receptor and macrophage-dependent pathways. In addition, it has been shown that variations in VWF glycosylation may play a role in the pathophysiology of type 1 VWD with accelerated VWF clearance. ${ }^{66}$ The p.Arg1205His variant (known as the Vicenza variant) is the classical mutation associated with accelerated VWF clearance which is characterized by a

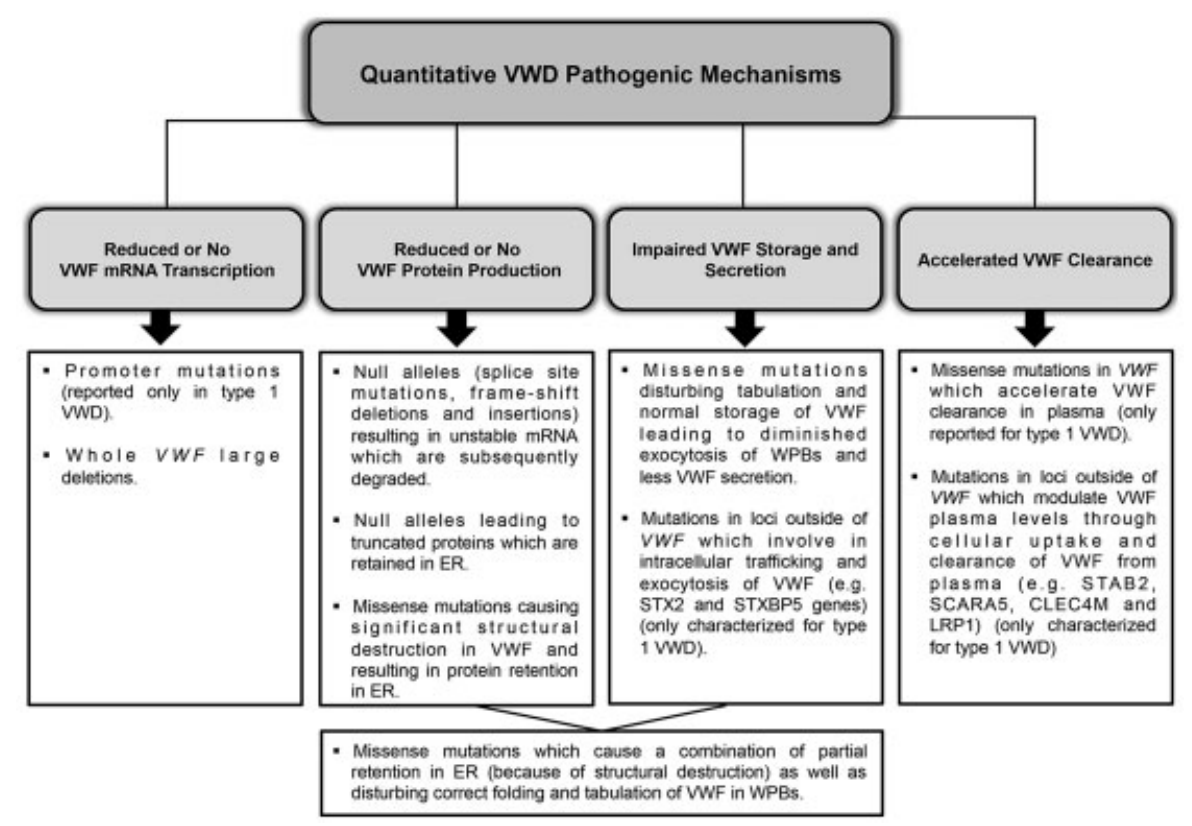

Fig. 2 Molecular mechanisms underlying quantitative VWF deficiencies. This diagram illustrates the main mechanisms through which production and biosynthesis of VWF are impaired. These mechanisms are described for both type 1 and type 3 VWD. Exceptions are VWF promoter mutations, mutations outside VWF involving intracellular trafficking of VWF and mutations associated with accelerated VWF clearance, which are only characterized for type 1 VWD. VWD, von Willebrand disease. 
significantly elevated VWFpp/VWF:Ag ratio (often >10) and slightly abnormal VWF multimer patterns. Recent studies have shown that enhanced clearance of the p.Arg1205His/Ser/Cys variants can be mediated through elevated binding to the receptors of hepatic and splenic macrophages. ${ }^{62}$ However, the pathogenic mechanisms of the other mutations involved in the etiology of the accelerated VWF phenotype are not yet clearly understood.

The most frequently occurring type 1 VWD mutation is $\mathrm{p}$. Tyr1584Cys substitution (in domain A2 VWF); it is usually detected as heterozygous in approximately $15 \%$ of index patients. The p.Tyr1584Cys variant is associated with VWF: Ag levels of approximately $40 \mathrm{IU} / \mathrm{dL}$, and it has demonstrated incomplete penetrance and variable bleeding severity. ${ }^{30,33,34}$ The in vitro studies and animal models have shown that $\mathrm{p}$. Tyr1584Cys resulted in impaired secretion and enhanced ADAMTS13 cleavage. ${ }^{67}$

\section{A VWF Mutation or a Functional Polymorphism?}

It is sometimes challenging to determine whether a certain sequence variant is disease-associated or is an interindividual difference with functional significance but no direct clinical relevance. The $V W F$ locus is highly polymorphic with a large number of single nucleotide polymorphisms (SNPs) in VWF coding sequences and promoter region (https://www.ncbi.nlm. nih.gov/projects/SNP/snp_ref.cgi?geneId=7450), some of which, in association with pathologic mutations or in specific haplotypes together with other variants, have an additive influence on VWF:Ag levels and affect VWD severity. ${ }^{68-70} \mathrm{~A}$ good example is the established association of linked polymorphic variations $\mathrm{C} / \mathrm{T}$ at $-1234, \mathrm{~A} / \mathrm{G}$ at -1185 , and $\mathrm{G} / \mathrm{A}$ at -1051 in the promoter region of $V W F$, which are segregated as two distinct haplotypes: $-1234 \mathrm{C} /-1185 \mathrm{~A} /-1051 \mathrm{G}$ (haplotype 1) and $-1234 \mathrm{~T} /-1185 \mathrm{G} /-1051 \mathrm{~A}$ (haplotype 2), with plasma VWF levels. Keightley et al showed that the homozygous individuals for haplotype 1 had the highest mean VWF:Ag levels $(0.962$ $\mathrm{U} / \mathrm{mL})$, intermediate values of $\mathrm{VWF}: \mathrm{Ag}(0.867 \mathrm{U} / \mathrm{mL})$ were found for heterozygotes, and homozygous subjects for haplotype 2 had the lowest mean plasma VWF:Ag levels $(0.776 \mathrm{U} / \mathrm{mL}){ }^{64}$ This association might be due to differential binding of transcription proteins regulating VWF expression to these haplotypes. $^{71}$

The Arg924Gln missense substitution is another frequent VWF coding region variant which is associated with low VWF: Ag levels in type $1 \mathrm{VWD}$ cohorts. It is recorded as type $1 /$ type $2 \mathrm{~N}$ VWD mutation in variant databases. However, an investigation including in vitro gene expression study and RNA transcript analysis of BOECs (blood outgrowth endothelial cells) isolated from a p.Arg924Gln carrier patient demonstrated that this substitution does not result in a major effect on VWF biosynthesis, but this polymorphism might be a polymorphic marker for an unknown intronic splicing variant, leading to activation of the $5^{\prime}$ cryptic splice-site within exon 28 of the $V W F .^{72}$

\section{Genetic Determinants Outside VWF Contributing to Variability in Plasma VWF Levels}

In the remaining $35 \%$ of type 1 VWD patients, for whom no causative mutation is identified in the coding region or proximal promoter of $V W F$, the genetic determinants might be found in deep intronic regions, a distant regulatory sequence of $V W F$ or at loci external to the VWF contributing to the VWF biosynthesis pathway. In recent years, genome-wide association studies, linkage analyses, and hypothesis-based in vitro and in vivo experiments have identified more than 10 novel loci (in addition to previously recognized $\mathrm{ABO}$ blood group genes and $V W F$ ) that are associated with variations in VWF plasma levels. These new gene determinants of VWF levels include genes coding STXBP5 (syntaxin-binding protein 5), SCARA5 (scavenger receptor class A-member 5), STAB2 (Stabilin-2), STX2 (syntaxin 2), TC2N (tandem C2 nuclear domains), CLEC4M (C-type lectin domain family 4-member M), UFM1 (ubiquitin-fold modifier 1), GPCR (platelet G-protein coupled receptor), AVPR2 (arginine vasopressin-2 receptor), LRP1 (lipoprotein receptor-related protein1), ACE (angiotensin-converting enzyme), and Siglec-5 (sialic acid binding Ig-like lectin 5). ${ }^{58,73-78}$ These loci may modulate VWF plasma levels either by involvement in intracellular trafficking and exocytosis of VWF (e.g., STX2 and STXBP5 genes) or through cellular uptake and clearance of VWF from plasma (e.g., STAB2, SCARA5, CLEC4M, and LRP1) ${ }^{78,79}$ Further ex vivo gene expression and in vivo mouse studies of CLEC4M provided additional evidence that a variable number of tandem repeat (VNTR) polymorphisms in CLEC4M gene influence VWF levels in plasma. The CLEC4M protein is a lectin receptor possessing an $\mathrm{N}$-terminal cytoplasmic domain, a transmembrane domain and an extracellular neck region which is highly polymorphic possessing three to nine VNTRs of a conserved 23-aa sequence. It has been demonstrated that CLEC4M functions as a VWF clearance receptor by binding and internalizing VWF and targeting it to early endosome. Furthermore, it was shown certain VNTR allele changes the affinity of CLEC4M binding, contributing to the variability of plasma VWF levels. ${ }^{80}$

\section{Pathogenic Mechanisms Underlying Type 3 VWD}

The genetic etiology of type 3 VWD is almost well defined, with a broad spectrum of mutations spread throughout the entire VWF which cause almost no production of VWF (VWF: $\mathrm{Ag}<5 \mathrm{IU} / \mathrm{dL}$ ). Type $3 \mathrm{VWD}$, which is inherited as a recessive disease, is caused due to either homozygous or compound heterozygous VWF mutations. However, it appears that 40 to $50 \%$ of type 3 VWD patients demonstrate codominant inheritance rather than a typical recessive inheritance pattern, which is acknowledged by heterozygous carriers having a mild type 1 VWD phenotype in their families. ${ }^{29,81}$ Type 3 VWD demonstrates variability in the severity of clinically relevant bleeding episodes, although VWF is virtually absent in all type 3 VWDs consistently. Assessment of the bleeding score (BS) in Canadian type 3 VWD cohorts demonstrated that patients with mutations within VWFpp exhibited more severe bleeding $(B S=22)$ than those with mutations elsewhere in VWF $(B S=13) .{ }^{29}$ Consistent with the aforementioned finding, it was recently shown that BS was significantly higher in type 3 patients with undetectable VWFpp $(B S=19.5)$ than in those with detectable VWFpp levels $(\mathrm{BS}=14){ }^{61}$ 
Genetic analysis of VWD type 3 cohorts from several populations has demonstrated that two variant VWF alleles (homozygous or compound heterozygous mutations) have been identified on average in approximately $85 \%$ of cases. $^{29,30,82-86}$ In the remaining $15 \%$ of the cases, either no causative variation was detected or only one VWF mutation was identified, which does not explain the severe phenotype of type 3 VWD.

Up to date, more than 320 sequence variations have been documented in HGMD (http://www.hgmd.cf.ac.uk/ac/gene. php?gene $=V W F$, accessed in March 2019), of which the majority $(\sim 80 \%)$ are null alleles comprising a miscellany of nonsense mutations ( 24\%), small deletions ( 15\%), splice-site variations ( $14 \%)$, small insertions ( $\sim 9 \%)$, partial and total VWF deletions ( $11 \%)$, large insertions/duplications ( $\sim 1 \%)$ as well as gene conversions ( 6\%; - Fig. 1C, D). An average of approximately $70 \%$ of the all sequence variations identified in cohort studies of type 3 VWD were reported for the first time, indicating the high susceptibility of $V W F$ for sequence variations and mutational heterogeneity in type $3.29,30,82,87,88$ Alloantibody development against VWF has been reported rarely, approximately 5 to $10 \%$ of type 3 VWD patients, and occurs most often in patients with nonsense mutations or partial/complete VWF deletions on both alleles. ${ }^{89-92}$

Nonsense mutations: More than 70 nonsense mutations are documented in HGMD which constitute the majority of null alleles in type 3 VWD. Nonsense mutations are scattered over the whole VWF from D1 to C domains of VWF protein with a high incidence at the $\mathrm{CPG}$ mutational hot spot (-Fig. 1D).

Small deletions and insertions: Several small deletions (about 50) and insertions (more than 50) resulting in a shift of the reading frame and consequently a premature translational stop codon have been documented for type 3 VWD. These small deletions and insertions were mainly located in a stretch of the same nucleotide (e.g., c.2435delC in a stretch of cytosine) or within a string of a dinucleotide (e.g., c.4092delAC in a stretch of ACACAC). ${ }^{30,83}$ The frameshift deletion of single cytosine in exon 18 of the VWF (c.2435delC, p.Pro812Argfs*31), which had been identified in the original VWD family from the Aland Islands, was found frequently in type 3 cohorts of European populations (German, Hungarian, Finish, Swedish, and Italian populations)..$^{30,83,87,93}$ However, the small deletion c.2435delC was not detected in investigated Canadian and Indian populations, ${ }^{29,84,92}$ whereas the small insertion c.8418_8419insTCCC (p.Pro2807Leufs* ${ }^{*} 4$ ) and the small deletion c.2908delC (p.Leu970Serfs ${ }^{*}$ ) are the most frequent type 3 frameshift mutation in Canadian and Indian populations, respectively. ${ }^{29,92}$

Splicing site variations: A notable number of putative splice site substitutions (more than 40 core splice-site variants) affecting consensus 3' acceptor and 5' donor splice sites have been recorded for type 3 VWD. However, the pathogenicity of only a few of them has been evaluated in detail by RNA transcript analyses. Using total RNA isolated from platelets and leukocytes, it is confirmed that the donor splice-site variant $533-2 A>G$ in intron 5 of $V W F$ and the acceptor splice-site substitution $8155+3 \mathrm{G}>\mathrm{C}$ in intron 50 impair recognition of consensus splicing signals and eventually resulted in skipping of exons 6 and 50, respectively. The acceptor splice site $7730-1 \mathrm{G}>\mathrm{C}$ in intron 45 of VWF activates a cryptic acceptor splice site in exon 46, two nucleotides downstream of the original splice. ${ }^{4}$ Another splice-site variant $G>T$ at position $7770+1$ in intron 46 caused a combination of different splicing defects including activation of a cryptic splice site in intron 46, 25 nucleotides downstream of the original donor core splice site, as well as skipping of exon $46 .{ }^{94}$ Eventually, these splicing defects change the reading frame of the protein leading to premature stop codons and consequently result in truncated proteins with no function. ${ }^{44}$ Alternatively, the splicing defects may lead to nonstable mRNA and subsequently nonsense-mediated mRNA decay (NMD). An example for this incident is the core acceptor splicing variant c.7082-2A $>C$ whose deleterious effect was hidden by NMD (no aberrant mRNA). Homozygosity of mRNA SNPs (which were detected as heterozygous at the genomic level) confirmed the absence of the transcript from the second allele and degradation of the abnormal mRNA (-Fig. 2). ${ }^{44}$

Large deletions: About 35 VWF large deletions encompassing (homozygous or compound heterozygous) deletions of either only one exon (exon 6 and exon 42 including some intronic regions), longer segments of the VWF affecting several exons (exons 1-3, 1-5, 4-5, 4-34, 4-49, 5-16, 6-16, 11-16, 14-15, 17-18, 14-52, 16-43, 16-52, 17-18, $19-20,17-25,22-43,23-52,26-34,33-34,33-38$, and $48-52$ ), or even complete VWF are described (several reports). ${ }^{29,82,92,95}$ The deletion of the entire VWF and deletion of exons $4-5$ have been frequently reported in different populations. ${ }^{29,30,96,97}$ Of interest, these two large deletions have also been detected in type 3 heterozygous family members (diagnosed as mild type $1 \mathrm{VWD}$ ). This suggests a higher incidence of large deletions in type $1 \mathrm{VWD}$ than it is thought. Genomic structure analysis of breakpoints has shown that deletion junctions of three large deletions of exons $4-5,1-3$, and 5-16 of VWF were flanked by homologous Alu repetitive elements, promoting unequal homologous recombination and Alu-mediated deletions. ${ }^{87,96}$ On the other hand, it seemed that complete VWF deletion detected in German and Italian populations was not driven by an Alu-mediated mechanism but rather by a nonhomologousend-joining-dependent DNA repair mechanism. ${ }^{97}$ The large deletions may lead to either no mRNA synthesis, changing the reading frame of a codon (out of frame) and retention of truncated protein in ER, or in- frame amino acids missing proteins which can interfere with the correct folding and biosynthesis of VWF. ${ }^{96}$ Using BOECs derived from a patient with deletion of exons 4-5VWF, it has been shown that a combination of reduced mRNA synthesis with ER retention of deleted VWF protein impairs VWF biosynthesis. ${ }^{98}$

Gene conversions: An interlocus gene conversion event between VWF on chromosome 12 (12p13.31) and VWF pseudogene on chromosome 22 (22q11.22 to 22q11.23) has been demonstrated as the cause of VWD type $3 .^{29,83,84}$ 
Gene conversions result from high homology between a pseudogene and the corresponding sequences in $V W F$ (exons 23-34). The reported gene conversions (more than 20) are characterized by multiple missense and nonsense substitutions within exons 23 to 34 of VWF (domains D3-A1) which are distributed in varying lengths between $<100$ to 3,000 bp. ${ }^{82,84,92}$ Gene conversions are particularly prevalent in Indian populations.

Missense substitutions: Missense variations comprise approximately $20 \%$ of sequence variations which are spread over the entire VWF, within SP and D1-CK domains -Table 2. ${ }^{29-31,82,92,99-101}$ Nonetheless, they are mainly accumulated within domains D1 and D2 of VWFpp ( $45 \%$ of missense substitutions; - Fig. 1D). The VWFpp missense mutations disrupt the conformation of VWFpp, which interrupt proper VWFpp/mature VWF alignment required for disulfide-linked multimers. ${ }^{102,103}$ Consequently, they cause significant intracellular retention of the protein and impaired secretion. ${ }^{104-106}$

\section{Conclusion}

The genetic basis of type 1 and type 3 VWD differs from each other. The characterized genetic VWF defects in both types 1 and 3 VWD are very heterogeneous, including point mutations, frameshift variations, transcriptional aberrations, and chromosomal rearrangements. Furthermore, these genetic variations are not confined to a specific region of the VWF, but they are spread throughout the whole VWF. The diagnostic genetic analysis of VWD, therefore, requires sequencing of all VWF exons and their surrounding intronic regions as well as analysis tests for gene rearrangement screening like MLPA.

Despite significant progress in our understanding of molecular mechanisms underlying quantitative VWF deficiencies, questions regarding genetic determinants affecting VWF levels in plasma remain to be answered, and genetic diagnosis of quantitative VWD is still challenging. The VWF is highly polymorphic, with a long list of reported SNPs detected in healthy populations, indicating difficulty in ascertaining the pathologic nature of the novel identified sequence substitutions and correlating them with the manifesting phenotype. Hence, further functional evaluations including in vitro gene expression studies, RNA transcript analysis, investigation biosynthesis and storage of VWF in BOECs isolated from the patients, and in vivo animal studies are required to confirm the contribution of these genetic variations to disease pathogenesis. About $35 \%$ of type 1 and 15\% of type 3 VWD cases remain without clear causative genetic defect within VWF. For the majority of type 3 mutation-negative cases, the causative mutations might be located within deep intronic regions or distant regulatory sequences, whereas in type 1 negative-mutation cases, the causative defects are expected outside of the VWF locus. However, additional studies are required to entirely clarify the contribution of genetic loci outside of VWF to type 1 VWD.
Table 2 List of missense mutations identified in type 3 VWD and their impact on VWF biosynthesis based on in vitro functional studies

\begin{tabular}{|c|c|c|}
\hline aa Exchange & Domain & $\begin{array}{l}\text { Functional characterization/ } \\
\text { impact on splicing }\end{array}$ \\
\hline p.Met1Val ${ }^{29}$ & SP & $\mathrm{n} / \mathrm{a}$ \\
\hline p.Gly19Arg ${ }^{33}$ & SP & $\begin{array}{l}\text { No impact on the expression of } \\
\text { VWF/putative impact on } \\
\text { splicing } 49\end{array}$ \\
\hline p.Arg34Gly ${ }^{107}$ & D1/VWD & $\mathrm{n} / \mathrm{a}$ \\
\hline p.Cys35 $\operatorname{Arg}^{87}$ & D1/VWD & $\mathrm{n} / \mathrm{a}$ \\
\hline p.Asp47His ${ }^{83}$ & D1/VWD & $\mathrm{n} / \mathrm{a}$ \\
\hline p.Gly79Val ${ }^{31}$ & D1/VWD & $\mathrm{n} / \mathrm{a}$ \\
\hline p.Arg81Gly ${ }^{87}$ & D1/VWD & $\mathrm{n} / \mathrm{a}$ \\
\hline p.Ser85Pro 83 & D1/VWD & $\mathrm{n} / \mathrm{a}$ \\
\hline p.Tyr87Ser ${ }^{106}$ & D1/VWD & Multimerization deficiency ${ }^{106}$ \\
\hline p.Asp141Asn ${ }^{83}$ & D1/VWD & $\mathrm{n} / \mathrm{a}$ \\
\hline p.Asp141Tyr ${ }^{83}$ & D1/VWD & Multimerization deficiency ${ }^{103}$ \\
\hline p.Gly142Asp ${ }^{101}$ & D1/VWD & $\mathrm{n} / \mathrm{a}$ \\
\hline p.Leu150Pro ${ }^{110}$ & D1/VWD & $\mathrm{n} / \mathrm{a}$ \\
\hline p.Leu150Gln 99 & D1/VWD & $\mathrm{n} / \mathrm{a}$ \\
\hline p.Gly160Arg ${ }^{92}$ & D1/VWD & $\mathrm{n} / \mathrm{a}$ \\
\hline p.Arg273Trp ${ }^{104}$ & $\mathrm{D} 1 / \mathrm{C} 8$ & Multimerization deficiency ${ }^{104}$ \\
\hline p.Arg273Pro ${ }^{101}$ & $\mathrm{D} 1 / \mathrm{C} 8$ & $\mathrm{n} / \mathrm{a}$ \\
\hline p.Cys275Ser ${ }^{91}$ & $\mathrm{D} 1 / \mathrm{C} 8$ & Multimerization deficiency ${ }^{102}$ \\
\hline p.Cys295Ser ${ }^{87}$ & $\mathrm{D} 1 / \mathrm{TIL}$ & $\mathrm{n} / \mathrm{a}$ \\
\hline p.Tyr301Cys ${ }^{31}$ & $\mathrm{D} 1 / \mathrm{TIL}$ & $\mathrm{n} / \mathrm{a}$ \\
\hline p.Asn318 Lys $^{92}$ & $\mathrm{D} 1 / \mathrm{TIL}$ & $\mathrm{n} / \mathrm{a}$ \\
\hline p.Lys355Arg ${ }^{82}$ & $\mathrm{D} 1 / \mathrm{E}$ & $\mathrm{n} / \mathrm{a}$ \\
\hline p.Trp377Cys 118 & $\mathrm{D} 1 / \mathrm{E}$ & $\mathrm{n} / \mathrm{a}$ \\
\hline p.Asp437Tyr ${ }^{82}$ & D2/VWD & $\mathrm{n} / \mathrm{a}$ \\
\hline p.Arg447Trp ${ }^{31}$ & D2/VWD & $\mathrm{n} / \mathrm{a}$ \\
\hline p.Cys584Phe 105 & $\mathrm{D} 2 / \mathrm{C} 8$ & $\begin{array}{l}\text { Impairing storage and } \\
\text { secretion } 105\end{array}$ \\
\hline p.His596Asn ${ }^{87}$ & $\mathrm{D} 2 / \mathrm{C} 8$ & $\mathrm{n} / \mathrm{a}$ \\
\hline p.Cys623Tyr ${ }^{87}$ & $\mathrm{D} 2 / \mathrm{C} 8$ & $\mathrm{n} / \mathrm{a}$ \\
\hline p.Cys633Arg ${ }^{82}$ & $\mathrm{D} 2 / \mathrm{C} 8$ & $\mathrm{n} / \mathrm{a}$ \\
\hline p.Met736lle & $\mathrm{D} 2 / \mathrm{E}$ & $\mathrm{n} / \mathrm{a}$ \\
\hline p.Met814lle 82 & $\mathrm{D}^{\prime} / \mathrm{IL}$ & $\mathrm{n} / \mathrm{a}$ \\
\hline p.cys827Tyr ${ }^{100}$ & $\mathrm{D}^{\prime} / \mathrm{IL}$ & $\mathrm{n} / \mathrm{a}$ \\
\hline p.Arg854Trp 92 & $\overline{D^{\prime} / E}$ & $\mathrm{n} / \mathrm{a}$ \\
\hline p.Gly967Val $^{101}$ & D3/VWD & $\mathrm{n} / \mathrm{a}$ \\
\hline p.Cys1071Phe ${ }^{83}$ & $\mathrm{D} 3 / \mathrm{C} 8$ & $\mathrm{n} / \mathrm{a}$ \\
\hline p.Tyr1107His ${ }^{101}$ & $\mathrm{D} 3 / \mathrm{C} 8$ & $\mathrm{n} / \mathrm{a}$ \\
\hline p.Cys $1165 \operatorname{Arg}^{103}$ & $\mathrm{D} 3 / \mathrm{C} 8$ & $\mathrm{n} / \mathrm{a}$ \\
\hline p.Val1279lle 92 & $\mathrm{~A} 1$ & $\mathrm{n} / \mathrm{a}$ \\
\hline p.Leu1288Val ${ }^{92}$ & $\mathrm{~A} 1$ & $\mathrm{n} / \mathrm{a}$ \\
\hline p.Arg1315Cys ${ }^{99}$ & $\mathrm{~A} 1$ & $\begin{array}{l}\text { A combination of retention in ER } \\
\text { along with defect in storage in } \\
\text { WPBs and secretion }\end{array}$ \\
\hline p.lle1509Val ${ }^{82}$ & $\mathrm{~A} 2$ & $\mathrm{n} / \mathrm{a}$ \\
\hline
\end{tabular}


Table 2 (Continued)

\begin{tabular}{|c|c|c|}
\hline aa Exchange & Domain & $\begin{array}{l}\text { Functional characterization/ } \\
\text { impact on splicing }\end{array}$ \\
\hline p.Gln1667His ${ }^{92}$ & $A 2-A 3$ & $\mathrm{n} / \mathrm{a}$ \\
\hline p.Glu1673Asp ${ }^{92}$ & $A 2-A 3$ & $\mathrm{n} / \mathrm{a}$ \\
\hline p.Gln1931 His ${ }^{92}$ & D4/D4N & $\mathrm{n} / \mathrm{a}$ \\
\hline p.Cys1946Phe ${ }^{101}$ & D4/D4N & $\mathrm{n} / \mathrm{a}$ \\
\hline p.Gly2044Asp ${ }^{82}$ & D4/VWD & $\mathrm{n} / \mathrm{a}$ \\
\hline p.Asn2066Ser ${ }^{110}$ & D4/VWD & $\mathrm{n} / \mathrm{a}$ \\
\hline p.Cys2174Gly ${ }^{83}$ & $\mathrm{D} 4 / \mathrm{C} 8$ & $\mathrm{n} / \mathrm{a}$ \\
\hline p.Cys2184Ser ${ }^{90}$ & $\mathrm{D} 4 / \mathrm{C} 8$ & $\begin{array}{l}\text { Intracellular retention, impaired } \\
\text { secretion }^{90}\end{array}$ \\
\hline p.Cys2184Phe ${ }^{31}$ & $\mathrm{D} 4 / \mathrm{C} 8$ & $\mathrm{n} / \mathrm{a}$ \\
\hline p.Cys 2212 Arg $^{90}$ & $\mathrm{D} 4 / \mathrm{TIL}$ & $\begin{array}{l}\text { Intracellular retention, impaired } \\
\text { secretion }^{90}\end{array}$ \\
\hline p.Cys2237Arg 29 & $\mathrm{D} 4 / \mathrm{TIL}$ & $\mathrm{n} / \mathrm{a}$ \\
\hline p.Cys 2283 Arg $^{30}$ & $\mathrm{C} 1$ & $\mathrm{n} / \mathrm{a}$ \\
\hline p.Cys 2325 Ser $^{90}$ & $\mathrm{C} 1$ & $\begin{array}{l}\text { Intracellular retention, impaired } \\
\text { secretion }^{90}\end{array}$ \\
\hline p.Cys2362Phe ${ }^{86}$ & $\mathrm{C2}$ & $\begin{array}{l}\text { Intracellular retention and im- } \\
\text { paired secretion } \\
86\end{array}$ \\
\hline p.Pro2373Leu ${ }^{101}$ & $\mathrm{C} 2$ & $\mathrm{n} / \mathrm{a}$ \\
\hline p.Cys2394Trp ${ }^{96}$ & $\mathrm{C2}$ & $\mathrm{n} / \mathrm{a}$ \\
\hline p.Thr2454Asn ${ }^{109}$ & $\mathrm{C3}$ & $\mathrm{n} / \mathrm{a}$ \\
\hline p.Cys2491Arg ${ }^{82}$ & $\mathrm{C3}$ & $\begin{array}{l}\text { Retention in ER and a strong } \\
\text { reduction in secretion }\end{array}$ \\
\hline p.Arg2535Gln 92 & $\mathrm{C} 4$ & $\mathrm{n} / \mathrm{a}$ \\
\hline p.Asn2546Tyr ${ }^{61}$ & $\mathrm{C} 4$ & $\mathrm{n} / \mathrm{a}$ \\
\hline p.Asn2636Tyr ${ }^{29}$ & $\mathrm{C5}$ & $\mathrm{n} / \mathrm{a}$ \\
\hline p.Cys2671 yyr $^{54}$ & C6 & $\begin{array}{l}\text { ER retention and reduced storage } \\
\text { in WPBs, impaired secretion }\end{array}$ \\
\hline p.Cys2739Tyr ${ }^{99}$ & CK & $\begin{array}{l}\text { ER retention; disorganized stor- } \\
\text { age of } V W F \text {, impaired secretion } \\
53\end{array}$ \\
\hline p.Cys2754Trp ${ }^{53}$ & CK & $\begin{array}{l}\text { ER retention; disorganized stor- } \\
\text { age of VWF, impaired secretion }{ }^{53}\end{array}$ \\
\hline p.Cys2804Tyr ${ }^{83}$ & CK & $\mathrm{n} / \mathrm{a}$ \\
\hline
\end{tabular}

Abbreviations: ER, endoplasmic reticulum; n/a: no answer; WPBs, Weibel-Palade bodies; VWF, von Willebrand factor.

Notes: List of type 3 missense mutations reported in the published literature with references. The location of the mutations on VWF domains and subdomains is based on updated domain designations. ${ }^{8}$ The impact of these missense variations on VWF biosynthesis (if reported) is given with references.

Lastly, improving our understanding of the pathogenesis mechanisms contributing to low VWF levels in plasma provides information to establish complementary molecular diagnostic tests and develop new treatment regiments for the quantitative trait of VWD.

\section{Recipient of the Günter Landbeck Award, 2016.}

\section{Conflict of Interest}

Dr. Oldenburg reports grants and personal fees from Bayer, grants and personal fees from Biotest, personal fees from Chugai, grants and personal fees from CSL
Behring, personal fees from Grifols, grants and personal fees from Novo Nordisk, grants and personal fees from Octapharma, personal fees from Pfizer, personal fees from Roche, personal fees from SOBI, grants and personal fees from Shire, outside the submitted work. Personal fees were received for travel support, participation in advisory boards, and participating in symposia as chair or speaker.

\section{References}

1 Leebeek FWG, Eikenboom JCJ. Von Willebrand's disease. N Engl J Med 2017;376(07):701-702

2 Goodeve AC. The genetic basis of von Willebrand disease. Blood Rev 2010;24(03):123-134

3 Sadler JE. von Willebrand factor: two sides of a coin. J Thromb Haemost 2005;3(08):1702-1709

4 Sadler JE, Budde U, Eikenboom JCJ, et al; Working Party on von Willebrand Disease Classification. Update on the pathophysiology and classification of von Willebrand disease: a report of the Subcommittee on von Willebrand factor. J Thromb Haemost 2006;4(10):2103-2114

5 Castaman G, Lethagen S, Federici AB, et al. Response to desmopressin is influenced by the genotype and phenotype in type 1 von Willebrand disease (VWD): results from the European Study MCMDM-1VWD. Blood 2008;111(07):3531-3539

6 Tischer A, Machha VR, Frontroth JP, et al. Enhanced local disorder in a clinically elusive von Willebrand factor provokes highaffinity platelet clumping. J Mol Biol 2017;429(14):2161-2177

7 Mancuso DJ, Tuley EA, Westfield LA, et al. Structure of the gene for human von Willebrand factor. J Biol Chem 1989;264(33): 19514-19527

8 Zhou Y-F, Eng ET, Zhu J, Lu C, Walz T, Springer TA. Sequence and structure relationships within von Willebrand factor. Blood 2012;120(02):449-458

9 Katsumi A, Tuley EA, Bodó I, Sadler JE. Localization of disulfide bonds in the cystine knot domain of human von Willebrand factor. J Biol Chem 2000;275(33):25585-25594

10 Voorberg J, Fontijn R, van Mourik JA, Pannekoek H. Domains involved in multimer assembly of von willebrand factor (vWF): multimerization is independent of dimerization. ЕMBO J 1990;9 (03):797-803

11 Purvis AR, Gross J, Dang LT, et al. Two Cys residues essential for von Willebrand factor multimer assembly in the Golgi. Proc Natl Acad Sci U S A 2007;104(40):15647-15652

12 Foster PA, Fulcher CA, Marti T, Titani K, Zimmerman TS. A major factor VIII binding domain resides within the amino-terminal 272 amino acid residues of von Willebrand factor. J Biol Chem 1987;262(18):8443-8446

13 Miyata S, Goto S, Federici AB, Ware J, Ruggeri ZM. Conformational changes in the A1 domain of von Willebrand factor modulating the interaction with platelet glycoprotein Ibalpha. J Biol Chem 1996;271(15):9046-9053

14 Pareti FI, Fujimura Y, Dent JA, Holland LZ, Zimmerman TS, Ruggeri ZM. Isolation and characterization of a collagen binding domain in human von Willebrand factor. J Biol Chem 1986;261 (32):15310-15315

15 Yuan Y, Zhang W, Yan R, et al. Identification of a novel 14-3-3zeta binding site within the cytoplasmic domain of platelet glycoprotein Ibalpha that plays a key role in regulating the von Willebrand factor binding function of glycoprotein Ib-IX. Circ Res 2009;105 (12):1177-1185

16 Jaffe EA, Hoyer LW, Nachman RL. Synthesis of von Willebrand factor by cultured human endothelial cells. Proc Natl Acad Sci U S A 1974;71(05):1906-1909

17 Sporn LA, Chavin SI, Marder VJ, Wagner DD. Biosynthesis of von Willebrand protein by human megakaryocytes. J Clin Invest 1985;76(03):1102-1106 
18 Zhou Y-F, Eng ET, Nishida N, Lu C, Walz T, Springer TA. A pHregulated dimeric bouquet in the structure of von Willebrand factor. EMBO J 2011;30(19):4098-4111

19 Vischer UM, Wagner DD. von Willebrand factor proteolytic processing and multimerization precede the formation of Weibel-Palade bodies. Blood 1994;83(12):3536-3544

20 Zhou Y-F, Springer TA. Highly reinforced structure of a C-terminal dimerization domain in von Willebrand factor. Blood 2014;123 (12):1785-1793

21 Lippok S, Kolšek K, Löf A, et al. von Willebrand factor is dimerized by protein disulfide isomerase. Blood 2016;127(09):1183-1191

22 Journet AM, Saffaripour S, Wagner DD. Requirement for both D domains of the propolypeptide in von Willebrand factor multimerization and storage. Thromb Haemost 1993;70(06): 1053-1057

23 Wise RJ, Pittman DD, Handin RI, Kaufman RJ, Orkin SH. The propeptide of von Willebrand factor independently mediates the assembly of von Willebrand multimers. Cell 1988;52(02): 229-236

24 Wagner DD, Olmsted JB, Marder VJ. Immunolocalization of von Willebrand protein in Weibel-Palade bodies of human endothelial cells. J Cell Biol 1982;95(01):355-360

25 Mayadas T, Wagner DD, Simpson PJ. von Willebrand factor biosynthesis and partitioning between constitutive and regulated pathways of secretion after thrombin stimulation. Blood 1989;73(03):706-711

26 Lopes da Silva M, Cutler DF. von Willebrand factor multimerization and the polarity of secretory pathways in endothelial cells. Blood 2016;128(02):277-285

27 Canis K, McKinnon TAJ, Nowak A, et al. Mapping the N-glycome of human von Willebrand factor. Biochem J 2012;447(02): 217-228

28 Solecka BA, Weise C, Laffan MA, Kannicht C. Site-specific analysis of von Willebrand factor O-glycosylation. J Thromb Haemost 2016;14(04):733-746

29 Bowman M, Tuttle A, Notley C, et al; Association of Hemophilia Clinic Directors of Canada. The genetics of Canadian type 3 von Willebrand disease: further evidence for co-dominant inheritance of mutant alleles. J Thromb Haemost 2013;11(03):512-520

30 Yadegari H, Driesen J, Pavlova A, Biswas A, Hertfelder HJ, Oldenburg J. Mutation distribution in the von Willebrand factor gene related to the different von Willebrand disease (VWD) types in a cohort of VWD patients. Thromb Haemost 2012;108 (04):662-671

31 Veyradier A, Boisseau P, Fressinaud E, et al; French Reference Center for von Willebrand disease. A laboratory phenotype/ genotype correlation of 1167 French patients from 670 families with von Willebrand disease: a new epidemiologic picture. Medicine (Baltimore) 2016;95(11):e3038

32 Flood VH, Christopherson PA, Gill JC, et al. Clinical and laboratory variability in a cohort of patients diagnosed with type $1 \mathrm{VWD}$ in the United States. Blood 2016;127(20):2481-2488

33 Goodeve A, Eikenboom J, Castaman G, et al. Phenotype and genotype of a cohort of families historically diagnosed with type 1 von Willebrand disease in the European study, Molecular and Clinical Markers for the Diagnosis and Management of Type 1 von Willebrand Disease (MCMDM-1VWD). Blood 2007;109 (01):112-121

34 James PD, Notley C, Hegadorn C, et al. The mutational spectrum of type 1 von Willebrand disease: results from a Canadian cohort study. Blood 2007;109(01):145-154

35 Daidone V, Gallinaro L, Grazia Cattini M, et al. An apparently silent nucleotide substitution (c.7056C $>\mathrm{T}$ ) in the von Willebrand factor gene is responsible for type 1 von Willebrand disease. Haematologica 2011;96(06):881-887

36 Hampshire DJ, Goodeve AC. The molecular basis of von Willebrand disease: the under investigated, the unexpected and the overlooked. Haematologica 2011;96(06):798-800
37 Gill JC, Endres-Brooks J, Bauer PJ, Marks WJ Jr, Montgomery RR. The effect of ABO blood group on the diagnosis of von Willebrand disease. Blood 1987;69(06):1691-1695

38 Gallinaro L, Cattini MG, Sztukowska M, et al. A shorter von Willebrand factor survival in O blood group subjects explains how $\mathrm{ABO}$ determinants influence plasma von Willebrand factor. Blood 2008;111(07):3540-3545

39 James PD, Lillicrap D. von Willebrand disease: clinical and laboratory lessons learned from the large von Willebrand disease studies. Am J Hematol 2012;87(Suppl 1):S4-S11

40 Budde U. Diagnosis of von Willebrand disease subtypes: implications for treatment. Haemophilia 2008;14(Suppl 5):27-38

41 Bodó I, Katsumi A, Tuley EA, Eikenboom JC, Dong Z, Sadler JE. Type 1 von Willebrand disease mutation Cys1149Arg causes intracellular retention and degradation of heterodimers: a possible general mechanism for dominant mutations of oligomeric proteins. Blood 2001;98(10):2973-2979

42 Castaman G, Eikenboom JC, Missiaglia E, Rodeghiero F. Autosomal dominant type 1 von willebrand disease due to G3639T mutation (C1130F) in exon 26 of von Willebrand factor gene: description of five Italian families and evidence for a founder effect. Br J Haematol 2000;108(04):876-879

43 Casonato A, Cattini MG, Barbon G, Daidone V, Pontara E. Severe, recessive type 1 is a discrete form of von Willebrand disease: the lesson learned from the c.1534-3C $>$ A von Willebrand factor mutation. Thromb Res 2015;136(03):682-686

44 Corrales I, Ramírez L, Altisent C, Parra R, Vidal F. The study of the effect of splicing mutations in von Willebrand factor using RNA isolated from patients' platelets and leukocytes. J Thromb Haemost 2011;9(04):679-688

45 Gallinaro L, Sartorello F, Pontara E, et al. Combined partial exon skipping and cryptic splice site activation as a new molecular mechanism for recessive type 1 von Willebrand disease. Thromb Haemost 2006;96(06):711-716

46 Hawke L, Bowman ML, Poon M-C, Scully MF, Rivard GE, James PD. Characterization of aberrant splicing of von Willebrand factor in von Willebrand disease: an underrecognized mechanism. Blood 2016;128(04):584-593

47 Berber E, Ozbil M, Brown C, Baslar Z, Caglayan SH, Lillicrap D. Functional characterisation of the type 1 von Willebrand disease candidate VWF gene variants: p.M771I, p.L881R and p.P1413L. Blood Transfus 2017;15(06):548-556

48 Castaman G, Giacomelli SH, Jacobi PM, et al. Reduced von Willebrand factor secretion is associated with loss of Weibel-Palade body formation. J Thromb Haemost 2012;10(05):951-958

49 Eikenboom J, Hilbert L, Ribba AS, et al. Expression of 14 von Willebrand factor mutations identified in patients with type 1 von Willebrand disease from the MCMDM-1VWD study. J Thromb Haemost 2009;7(08):1304-1312

50 Groeneveld DJ, Wang J-W, Mourik MJ, et al. Storage and secretion of naturally occurring von Willebrand factor A domain variants. Br J Haematol 2014;167(04):529-540

51 Schneppenheim R, Budde U, Batlle J, et al. Intracellular retention, enhanced clearance, and defective FVIII binding are common features of Von Willebrand factor D'-D3 domain mutations in patients with Von Willebrand disease type 1 from the European Mcmdm-1VWD study. Blood 2012;120(21):99

52 Shapiro SE, Nowak AA, Wooding C, Birdsey G, Laffan MA, McKinnon TA. The von Willebrand factor predicted unpaired cysteines are essential for secretion. J Thromb Haemost 2014;12 (02):246-254

53 Wang J-W, Valentijn KM, de Boer HC, et al. Intracellular storage and regulated secretion of von Willebrand factor in quantitative von Willebrand disease. J Biol Chem 2011;286(27):24180-24188

54 Wang J-W, Groeneveld DJ, Cosemans G, et al. Biogenesis of Weibel-Palade bodies in von Willebrand's disease variants with impaired von Willebrand factor intrachain or interchain disulfide bond formation. Haematologica 2012;97(06):859-866 
55 Wang J-W, Bouwens EAM, Pintao MC, et al. Analysis of the storage and secretion of von Willebrand factor in blood outgrowth endothelial cells derived from patients with von Willebrand disease. Blood 2013;121(14):2762-2772

56 White-Adams TC, Ng CJ, Jacobi PM, Haberichter SL, Di Paola JA Mutations in the D'D3 region of VWF traditionally associated with type $1 \mathrm{VWD}$ lead to quantitative and qualitative deficiencies of VWF. Thromb Res 2016;145:112-118

57 Yadegari H, Driesen J, Pavlova A, et al. Insights into pathological mechanisms of missense mutations in C-terminal domains of von Willebrand factor causing qualitative or quantitative von Willebrand disease. Haematologica 2013;98(08):1315-1323

58 Casari C, Lenting PJ, Wohner N, Christophe OD, Denis CV. Clearance of von Willebrand factor. J Thromb Haemost 2013; 11(Suppl 1):202-211

59 Haberichter SL, Balistreri M, Christopherson P, et al. Assay of the von Willebrand factor (VWF) propeptide to identify patients with type 1 von Willebrand disease with decreased VWF survival. Blood 2006;108(10):3344-3351

60 Millar CM, Riddell AF, Brown SA, et al. Survival of von Willebrand factor released following DDAVP in a type 1 von Willebrand disease cohort: influence of glycosylation, proteolysis and gene mutations. Thromb Haemost 2008;99(05):916-924

61 Sanders YV, Groeneveld D, Meijer K, et al; WiN study group. von Willebrand factor propeptide and the phenotypic classification of von Willebrand disease. Blood 2015;125(19):3006-3013

62 Rawley O, O'Sullivan JM, Chion A, et al. von Willebrand factor arginine 1205 substitution results in accelerated macrophagedependent clearance in vivo. J Thromb Haemost 2015;13(05): 821-826

63 Schooten CJ, Tjernberg P, Westein E, et al. Cysteine-mutations in von Willebrand factor associated with increased clearance. J Thromb Haemost 2005;3(10):2228-2237

64 Haberichter SL, Castaman G, Budde U, et al. Identification of type 1 von Willebrand disease patients with reduced von Willebrand factor survival by assay of the VWF propeptide in the European study: molecular and clinical markers for the diagnosis and management of type 1 VWD (MCMDM-1VWD). Blood 2008; 111(10):4979-4985

65 Chion A, O'Sullivan JM, Drakeford C, et al. N-linked glycans within the A2 domain of von Willebrand factor modulate macrophagemediated clearance. Blood 2016;128(15):1959-1968

66 O'Sullivan JM, Aguila S, McRae E, et al. N-linked glycan truncation causes enhanced clearance of plasma-derived von Willebrand factor. J Thromb Haemost 2016;14(12):2446-2457

67 Pruss CM, Golder M, Bryant A, et al. Pathologic mechanisms of type 1 VWD mutations R1205H and Y1584C through in vitro and in vivo mouse models. Blood 2011;117(16):4358-4366

68 Wang QY, Song J, Gibbs RA, Boerwinkle E, Dong JF, Yu FL. Characterizing polymorphisms and allelic diversity of von Willebrand factor gene in the 1000 genomes. J Thromb Haemost 2013;11(02): 261-269

69 Daidone V, Pontara E, Romualdi C, et al. Microsatellite (GT)(n) is part of the von Willebrand factor (VWF) promoter region that influences the glucocorticoid-induced increase in VWF in Cushing's syndrome. Thromb Res 2010;125(06):e275-e280

70 Di Bitondo R, Cameron CL, Daly ME, et al. The $-1185 \mathrm{~A} / \mathrm{G}$ and -1051 G/A dimorphisms in the von Willebrand factor gene promoter and risk of myocardial infarction. $\mathrm{Br} \mathrm{J}$ Haematol 2001;115(03):701-706

71 Keightley AM, Lam YM, Brady JN, Cameron CL, Lillicrap D. Variation at the von Willebrand factor (vWF) gene locus is associated with plasma vWF:Ag levels: identification of three novel single nucleotide polymorphisms in the vWF gene promoter. Blood 1999;93(12):4277-4283

72 Berber E, James PD, Hough C, Lillicrap D. An assessment of the pathogenic significance of the R924Q von Willebrand factor substitution. J Thromb Haemost 2009;7(10):1672-1679
73 Antoni G, Oudot-Mellakh T, Dimitromanolakis A, et al. Combined analysis of three genome-wide association studies on vWF and FVIII plasma levels. BMC Med Genet 2011;12:102

74 Sanders YV, van der Bom JG, Isaacs A, et al; WiN Study Group. CLEC4M and STXBP5 gene variations contribute to von Willebrand factor level variation in von Willebrand disease. J Thromb Haemost 2015;13(06):956-966

75 Smith NL, Chen M-H, Dehghan A, et al; Wellcome Trust Case Control Consortium. Novel associations of multiple genetic loci with plasma levels of factor VII, factor VIII, and von Willebrand factor: the CHARGE (Cohorts for Heart and Aging Research in Genome Epidemiology) consortium. Circulation 2010;121(12):1382-1392

76 Stockley J, Nisar SP, Leo VC, et al; GAPP Study in Collaboration with the MCMDM-1VWD Study Group. Identification and characterization of novel variations in platelet G-protein coupled receptor (GPCR) genes in patients historically diagnosed with type 1 von Willebrand disease. PLoS One 2015;10(12):e0143913

77 van Loon JE, Sanders YV, de Wee EM, Kruip MJ, de Maat MP, Leebeek FW. Effect of genetic variation in STXBP5 and STX2 on von Willebrand factor and bleeding phenotype in type 1 von Willebrand disease patients. PLoS One 2012;7(07):e40624

78 van Loon J, Dehghan A, Weihong T, et al. Genome-wide association studies identify genetic loci for low von Willebrand factor levels. Eur J Hum Genet 2016;24(07):1096

79 Lillicrap D. Syntaxin-binding protein 5 exocytosis regulation: differential role in endothelial cells and platelets. J Clin Invest 2014;124(10):4231-4233

80 Rydz N, Swystun LL, Notley C, et al. The C-type lectin receptor CLEC4M binds, internalizes, and clears von Willebrand factor and contributes to the variation in plasma von Willebrand factor levels. Blood 2013;121(26):5228-5237

81 Castaman G, Rodeghiero F, Tosetto A, et al. Hemorrhagic symptoms and bleeding risk in obligatory carriers of type 3 von Willebrand disease: an international, multicenter study. J Thromb Haemost 2006;4(10):2164-2169

82 Ahmad F, Budde U, Jan R, et al. Phenotypic and molecular characterisation of type 3 von Willebrand disease in a cohort of Indian patients. Thromb Haemost 2013;109(04):652-660

83 Baronciani L, Cozzi G, Canciani MT, et al. Molecular defects in type 3 von Willebrand disease: updated results from 40 multiethnic patients. Blood Cells Mol Dis 2003;30(03):264-270

84 Gupta PK, Saxena R, Adamtziki E, et al. Genetic defects in von Willebrand disease type 3 in Indian and Greek patients. Blood Cells Mol Dis 2008;41(02):219-222

85 Sutherland MS, Keeney S, Bolton-Maggs PH, Hay CR, Will A, Cumming AM. The mutation spectrum associated with type 3 von Willebrand disease in a cohort of patients from the north west of England. Haemophilia 2009;15(05):1048-1057

86 Tjernberg P, Castaman G, Vos HL, Bertina RM, Eikenboom JC. Homozygous $\mathrm{C} 2362 \mathrm{~F}$ von Willebrand factor induces intracellular retention of mutant von Willebrand factor resulting in autosomal recessive severe von Willebrand disease. $\mathrm{Br} \mathrm{J}$ Haematol 2006;133(04):409-418

87 Mohl A, Boda Z, Jager R, et al. Common large partial VWF gene deletion does not cause alloantibody formation in the Hungarian type 3 von Willebrand disease population. J Thromb Haemost 2011;9(05):945-952

88 Corrales I, Ramírez L, Ayats J, Altisent C, Parra R, Vidal F. Integration of molecular and clinical data of 40 unrelated von Willebrand Disease families in a Spanish locus-specific mutation database: first release including 58 mutations. Haematologica 2010;95(11):1982-1984

89 James PD, Lillicrap D, Mannucci PM. Alloantibodies in von Willebrand disease. Blood 2013;122(05):636-640

90 Solimando M, Baronciani L, La Marca S, et al. Molecular characterization, recombinant protein expression, and mRNA analysis of type 3 von Willebrand disease: Studies of an Italian cohort of 10 patients. Am J Hematol 2012;87(09):870-874 
91 Baronciani L, Cozzi G, Canciani MT, et al. Molecular characterization of a multiethnic group of 21 patients with type 3 von Willebrand disease. Thromb Haemost 2000;84(04):536-540

92 Kasatkar P, Shetty S, Ghosh K. Genetic heterogeneity in a large cohort of Indian type 3 von Willebrand disease patients. PLoS One 2014;9(03):e92575

93 Jokela V, Lassila R, Szanto T, et al. Phenotypic and genotypic characterization of 10 Finnish patients with von Willebrand disease type 3: discovery of two main mutations. Haemophilia 2013;19(06):e344-e348

94 Castaman G, Platè M, Giacomelli SH, Rodeghiero F, Duga S. Alterations of mRNA processing and stability as a pathogenic mechanism in von Willebrand factor quantitative deficiencies. J Thromb Haemost 2010;8(12):2736-2742

95 Yadegari H, Driesen J, Hass M, Budde U, Pavlova A, Oldenburg J. Large deletions identified in patients with von Willebrand disease using multiple ligation-dependent probe amplification. J Thromb Haemost 2011;9(05):1083-1086

96 Sutherland MS, Cumming AM, Bowman M, et al. A novel deletion mutation is recurrent in von Willebrand disease types 1 and 3. Blood 2009;114(05):1091-1098

97 Schneppenheim R, Castaman G, Federici AB, et al. A common 253$\mathrm{kb}$ deletion involving VWF and TMEM16B in German and Italian patients with severe von Willebrand disease type 3. J Thromb Haemost 2007;5(04):722-728

98 Starke RD, Paschalaki KE, Dyer CEF, et al. Cellular and molecular basis of von Willebrand disease: studies on blood outgrowth endothelial cells. Blood 2013;121(14):2773-2784

99 Zhang ZP, Blombäck M, Egberg N, Falk G, Anvret M. Characterization of the von Willebrand factor gene (VWF) in von Willebrand disease type III patients from 24 families of Swedish and Finnish origin. Genomics 1994;21(01):188-193

100 Ouyang W, Yu Z, Yin J, Su J, Yang C, Ruan C. Two novel mutations identified in a type 3 von Willebrand disease patient. Blood Coagul Fibrinolysis 2014;25(08):909-911

101 Borràs N, Batlle J, Pérez-Rodríguez A, et al. Molecular and clinical profile of von Willebrand disease in Spain (PCM-EVW-ES): comprehensive genetic analysis by next-generation sequencing of 480 patients. Haematologica 2017;102(12):2005-2014

102 Baronciani L, Federici AB, Cozzi G, et al. Expression studies of missense mutations p.D141Y, p.C275S located in the propeptide of von Willebrand factor in patients with type 3 von Willebrand disease. Haemophilia 2008;14(03):549-555

103 Yin J, Ma Z, Su J, et al. Mutations in the D1 domain of von Willebrand factor impair their propeptide-dependent multimerization, intracellular trafficking and secretion. J Hematol Oncol 2015;8:73

104 Allen S, Abuzenadah AM, Hinks J, et al. A novel von Willebrand disease-causing mutation (Arg273Trp) in the von Willebrand factor propeptide that results in defective multimerization and secretion. Blood 2000;96(02):560-568
105 Daidone V, Barbon G, Pontara E, et al. Loss of cysteine 584 impairs the storage and release, but not the synthesis of von Willebrand factor. Thromb Haemost 2014;112(06):1159-1166

106 Rosenberg JB, Haberichter SL, Jozwiak MA, et al. The role of the D1 domain of the von Willebrand factor propeptide in multimerization of VWF. Blood 2002;100(05):1699-1706

107 Hampshire DJ, Abuzenadah AM, Cartwright A, et al. Identification and characterisation of mutations associated with von Willebrand disease in a Turkish patient cohort. Thromb Haemost 2013;110(02):264-274

108 Robertson JD, Yenson PR, Rand ML, et al. Expanded phenotypegenotype correlations in a pediatric population with type 1 von Willebrand disease. J Thromb Haemost 2011;9(09):1752-1760

109 Liang Q, Qin H, Ding Q, et al. Molecular and clinical profile of VWD in a large cohort of Chinese population: application of next generation sequencing and $\mathrm{CNV} \mathrm{Ple}^{\circledR}$ technique. Thromb Haemost 2017;117(08):1534-1548

110 Corrales I, Ramírez L, Altisent C, Parra R, Vidal F. Rapid molecular diagnosis of von Willebrand disease by direct sequencing. Detection of 12 novel putative mutations in VWF gene. Thromb Haemost 2009;101(03):570-576

111 Casonato A, Gallinaro L, Cattini MG, et al. Type 1 von Willebrand disease due to reduced von Willebrand factor synthesis and/or survival: observations from a case series. Transl Res 2010;155 (04):200-208

112 Casaña P, Martínez F, Haya S, Tavares A, Aznar JA. New mutations in exon 28 of the von Willebrand factor gene detected in patients with different types of von Willebrand's disease. Haematologica 2001;86(04):414-419

113 Holmberg L, Dent JA, Schneppenheim R, Budde U, Ware J, Ruggeri $Z M$. von Willebrand factor mutation enhancing interaction with platelets in patients with normal multimeric structure. J Clin Invest 1993;91(05):2169-2177

114 Melo-Nava BM, Benítez H, Palacios JJ, et al. Molecular study of VWF gene from Mexican Mestizo patients with von Willebrand disease, and the finding of three new mutations. Blood Cells Mol Dis 2007;39(03):361-365

115 Johansson AM, Halldén C, Säll T, Lethagen S. Variation in the VWF gene in Swedish patients with type 1 von Willebrand disease. Ann Hum Genet 2011;75(04):447-455

116 Johnsen JM, Auer PL, Morrison AC, et al; NHLBI Exome Sequencing Project. Common and rare von Willebrand factor (VWF) coding variants, VWF levels, and factor VIII levels in African Americans: the NHLBI Exome Sequencing Project. Blood 2013; 122(04):590-597

117 Lavin M, Aguila S, Schneppenheim S, et al. Novel insights into the clinical phenotype and pathophysiology underlying low VWF levels. Blood 2017;130(21):2344-2353

118 Schneppenheim R, Krey S, Bergmann F, et al. Genetic heterogeneity of severe von Willebrand disease type III in the German population. Hum Genet 1994;94(06):640-652 